\title{
A Novel Second-Generation EP2 Receptor Antagonist Reduces Neuroinflammation and Gliosis After Status Epilepticus in Rats
}

\author{
Asheebo Rojas ${ }^{1} \cdot$ Radhika Amaradhi $^{1}$ - Avijit Banik ${ }^{1}$. Chunxiang Jiang ${ }^{1} \cdot$ JuanMartin Abreu-Melon ${ }^{1} \cdot$ Sarah Wang $^{1}$. \\ Raymond Dingledine $^{1} \cdot$ Thota Ganesh ${ }^{1}$
}

Accepted: 7 November 2020 / Published online: 6 January 2021

(C) The American Society for Experimental NeuroTherapeutics, Inc. 2021

\begin{abstract}
Prostaglandin- $\mathrm{E}_{2}\left(\mathrm{PGE}_{2}\right)$, an important mediator of inflammation, achieves its functions via four different $\mathrm{G}$ protein-coupled receptors (EP1, EP2, EP3, and EP4). We previously demonstrated that the EP2 receptor plays a proinflammatory and neurodegenerative role after status epilepticus (SE). We recently developed TG8-260 as a second-generation highly potent and selective EP2 antagonist. Here, we investigate whether TG8-260 is anti-inflammatory and combats neuropathology caused by pilocarpineinduced SE in rats. Adult male Sprague-Dawley rats were injected subcutaneously with pilocarpine ( $380-400 \mathrm{mg} / \mathrm{kg})$ to induce SE. Following $60 \mathrm{~min}$ of SE, the rats were administered three doses of TG8-260 or vehicle and were allowed to recover. Neurodegeneration, neuroinflammation, gliosis, and blood-brain barrier (BBB) integrity were examined 4 days after SE. The results confirmed that pilocarpine-induced SE results in hippocampal neurodegeneration and a robust inflammatory response that persists days after SE. Furthermore, inhibition of the EP2 receptor by TG8-260 administered beginning $2 \mathrm{~h}$ after SE significantly reduced hippocampal neuroinflammation and gliosis but, in distinction to the earlier generation EP2 antagonist, did not mitigate neuronal injury or BBB breakdown. Thus, attenuation of neuroinflammation and gliosis is a common feature of EP2 inhibition following SE.
\end{abstract}

Key Words Pilocarpine $\cdot \mathrm{PGE}_{2} \cdot \mathrm{EP} 2 \cdot \mathrm{COX}-2 \cdot \mathrm{TG} 8-260 \cdot$ hippocampus $\cdot$ neurodegeneration $\cdot$ inflammation $\cdot$ status epilepticus $\cdot$ gliosis $\cdot$ BBB.

$\begin{array}{ll}\text { Abbreviations } \\ \text { PGE }_{2} & \text { Prostaglandin- } \mathrm{E}_{2} \\ \mathrm{EP} 2 & \text { Prostaglandin- } \mathrm{E}_{2} \text { receptor } 2 \\ \mathrm{EC}_{50} & \text { Half maximal effective concentration } \\ \mathrm{SE} & \text { Status epilepticus } \\ \text { GFAP } & \text { Glial fibrillary acidic protein } \\ \text { CA1 } & \text { Cornu ammonis } 1 \\ \text { CA3 } & \text { Cornu ammonis } 3 \\ \text { CT } & \text { Cycle threshold } \\ \text { Con } & \text { Control } \\ \text { COX-2 } & \text { Cyclooxygenase-2 } \\ \text { COX-1 } & \text { Cyclooxygenase-1 }\end{array}$

Asheebo Rojas

arajas@emory.edu

Thota Ganesh

tganesh@emory.edu

1 Department of Pharmacology and Chemical Biology, Emory University, 1510 Clifton Road NE, Atlanta, GA 30322, USA

$\begin{array}{ll}\text { pilo } & \text { Pilocarpine } \\ \text { IP } & \text { Intraperitoneal } \\ \text { SC } & \text { Subcutaneous } \\ \text { PO } & \text { Per oral } \\ \text { FJB } & \text { FluoroJade B } \\ \text { q R T - } & \text { Quantitative real-time polymerase chain reaction } \\ \text { PCR } & \\ \text { IL-1 } \beta & \text { Interleukin-1 } \beta \\ \text { TNF } \alpha & \text { Tumor necrosis factor alpha } \\ \text { CXCL10 } & \text { C-X-C motif chemokine 10 } \\ \text { CCL2 } & \text { Chemokine (C-C motif) ligand 2 } \\ \text { CCL3 } & \text { Chemokine (C-C motif) ligand 3 } \\ \text { CCL4 } & \text { Chemokine (C-C motif) ligand 4 } \\ \text { IL-6 } & \text { Interleukin 6 } \\ \text { GAPDH } & \text { Glyceraldehyde 3-phosphate dehydrogenase } \\ \text { HPRT1 } & \text { Hypoxanthine phosphoribosyltransferase 1 } \\ \text { Veh } & \text { Vehicle } \\ \text { mpk } & \text { mg/kg } \\ \text { TG8-260 } & \text { Potent and selective EP2 receptor antagonist } \\ \text { BBB } & \text { Blood-brain barrier }\end{array}$




\section{Introduction}

Status epilepticus (SE, an unremitting seizure or a series of seizures lasting longer than $5 \mathrm{~min}$ ) is a medical emergency. Survivors of SE can develop brain injury and long-term cognitive deficits. In rodent models of SE used in our laboratory, survivors exhibit weight loss, neuroinflammation, neurodegeneration, long-term cognitive deficits, and the development of spontaneous recurrent seizures (SRS) [1-9]. One of the goals of our research group is to combat neuropathologies associated with SE by selectively targeting the EP2 receptor. Exploring the role of the EP2 receptor in seizure-induced neuropathology is a novel approach that grew out of microarray studies of early epileptogenesis [10], which pointed to a multifaceted inflammatory response in the brain of rodents that experienced SE. This inflammatory response included induction of cyclooxygenase-2 (COX-2), a key enzyme involved in inflammation and pain. The cyclooxygenase enzymes are critical for the production of prostaglandin- $\mathrm{E}_{2}\left(\mathrm{PGE}_{2}\right)$ and other prostanoids, which are hormone-like substances that participate in many physiological functions. In particular, $\mathrm{PGE}_{2}$ promotes its actions via four different $G$ protein-coupled receptors (EP1, EP2, EP3, and EP4). These prostanoid receptors present alternative, more selective targets for drug development and disease modification in epilepsy compared to COX2 inhibitors, which display mixed efficacy and potentially harmful or fatal side effects $[11,12]$.

Inhibition of the EP2 receptor with a first-generation smallmolecule antagonist (TG6-10-1) developed by our group afforded neuroprotection after SE caused by pilocarpine, diisopropylfluorophosphate (DFP), and kainate in adult mice and rats, associated with reduced inflammation, accelerated functional recovery, and reduced cognitive deficits $[2,4,5$, 13]. Thus, inhibition of the EP 2 receptor could be a novel strategy for neuroprotection and functional restoration after brain injury in adults. The research lead compound, TG6-101 , is highly brain permeable but suffers from low aqueous solubility, the presence of an acryl amide ( $a, \beta$-enone) structural moiety that forms glutathione adducts, and only a 10-fold selectivity versus its closest prostanoid receptor, DP1. A continued medicinal chemistry and lead optimization campaign to overcome these deficiencies led to the identification of a second-generation EP2 receptor antagonist, TG8-260. Here, we tested the hypothesis that EP2 receptor inhibition by this highly selective EP2 antagonist, which has limited brain permeability, reduces neuropathology manifested by pilocarpineinduced SE in adult rats. We ask the questions: How selective is TG8-260 for EP2 receptors? What are the pharmacokinetic properties of TG8-260? Does TG8-260 oppose SE-induced neuropathology? To address these questions we used in vitro receptor and enzyme assays together with an adult rat model of SE produced by pilocarpine administration. The results support further investigation into the use of a selective EP2 antagonist as adjunct therapy for neuronal hyperexcitation, SE, and epilepsy.

\section{Methods}

\section{Ethics Statement}

All procedures concerning animals were approved by the Emory University Animal Care and Use Committee and conformed to the guidelines of the National Institutes of Health.

\section{Pilocarpine-Induced SE}

Status epilepticus (SE) was induced in adult male SpragueDawley rats (260-397 g body weight). To improve survival rats were injected with methylscopolamine and terbutaline $(2.5 \mathrm{mg} / \mathrm{kg}$ each, dissolved in a $0.9 \%$ saline solution) subcutaneously. After $30 \mathrm{~min}$, the rats were injected with pilocarpine hydrochloride $(380-400 \mathrm{mg} / \mathrm{kg}$, dissolved in a $0.9 \%$ saline solution) or an equivalent volume of $0.9 \%$ saline subcutaneously. In rats, pilocarpine-induced seizures consisted of distinct motor behaviors including tail extension, forelimb clonus, rearing, and falling. Animals presenting these behaviors with increased seizure intensity, duration, and frequency shortly after the injection of pilocarpine were in SE. The seizure activity was scored and recorded every $5 \mathrm{~min}$ for at least $1 \mathrm{~h}$ using a modified Racine scale [14] that is appropriate for scoring rat seizure behavior in our pilocarpine model (see below). Diazepam (10 mg/kg, i.p.; Hospira) was administered to interrupt SE after 60 min for experiments with TG8-260 (Fig. 1). For experiments investigating a 24-h neuropathology, a separate cohort of rats experienced SE for $90 \mathrm{~min}$ followed by administration of sodium pentobarbital $(25 \mathrm{mg} / \mathrm{kg}$, dissolved in $0.9 \%$ saline; Akorn Pharmaceuticals) subcutaneously to terminate SE (Fig. 2). Sodium pentobarbital was used in the 24-h experiments because diazepam fails to terminate SE lasting longer than $60 \mathrm{~min}$. After termination of SE, the rats were then placed individually into clean plastic cages with fresh bedding, soft food, and water and allowed to recover overnight. To maintain hydration, a single injection of lactated Ringer's solution ( $2 \mathrm{ml}$, i.p.) was administered when the rats were placed into cages. A modified Irwin test [15] was performed to access the health of rats prior to and after pilocarpine-induced SE as previously described $[4,5]$. The test was given three times (once prior to pilocarpine, $24 \mathrm{~h}$ after SE onset, and again just prior to euthanization) to observe physical or behavioral parameters: ptosis, exophthalmia, lacrimation, body posture, running versus walking (response to startle), hypoactivity versus hyperactivity, aggression, muscle tone, and vocalization when handled. Each observation item was scored as follows: $0=$ normal; $1=$ mild to moderate 
Fig. 1 Prolonged neuronal activation induced by pilocarpine. (A) Experimental paradigm of chemical administration in a rat model of pilocarpine-induced status epilepticus. (B) Fluorescent images taken from the hippocampus $(50 \times$ magnification) that reveal positive $\Delta$ Fosb staining in neurons in rats that experienced SE $24 \mathrm{~h}$ earlier but not in rats that did not experience status epilepticus. (C) $\Delta$ Fosb induction is also prominent $24 \mathrm{~h}$ after SE onset in the amygdala and piriform cortex (PC), but not in rats that did not experience SE. The images shown are representative of five sections each from at least three rats. Scale bar, $500 \mu \mathrm{m}$
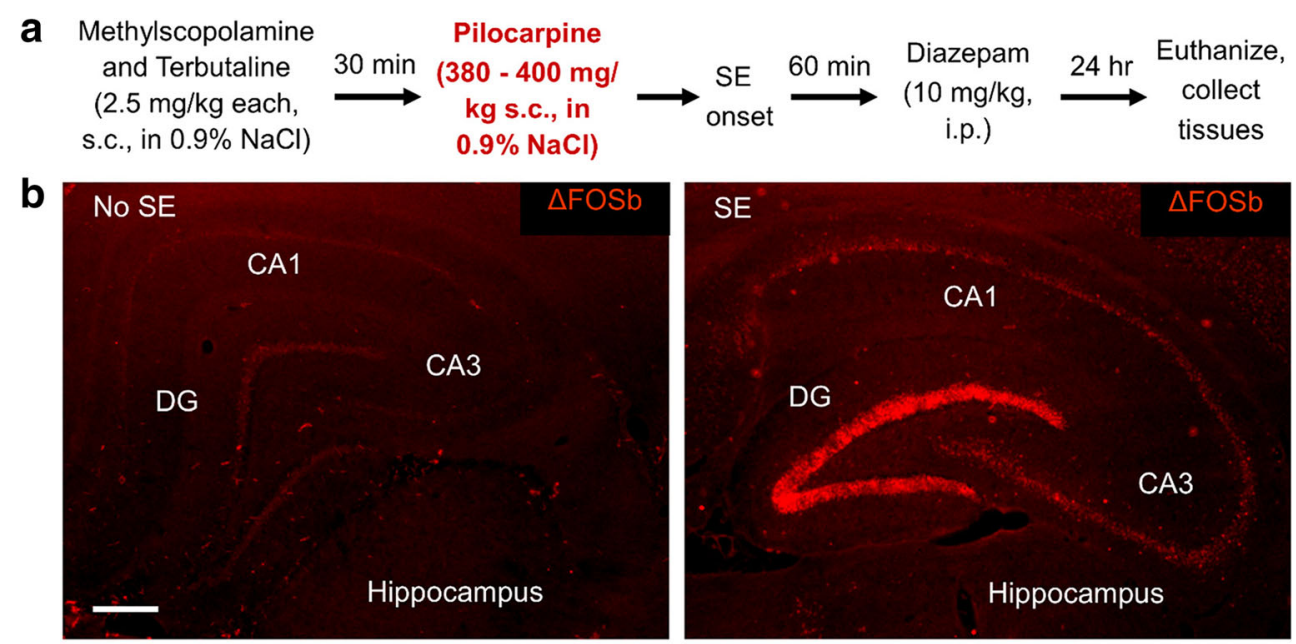

C
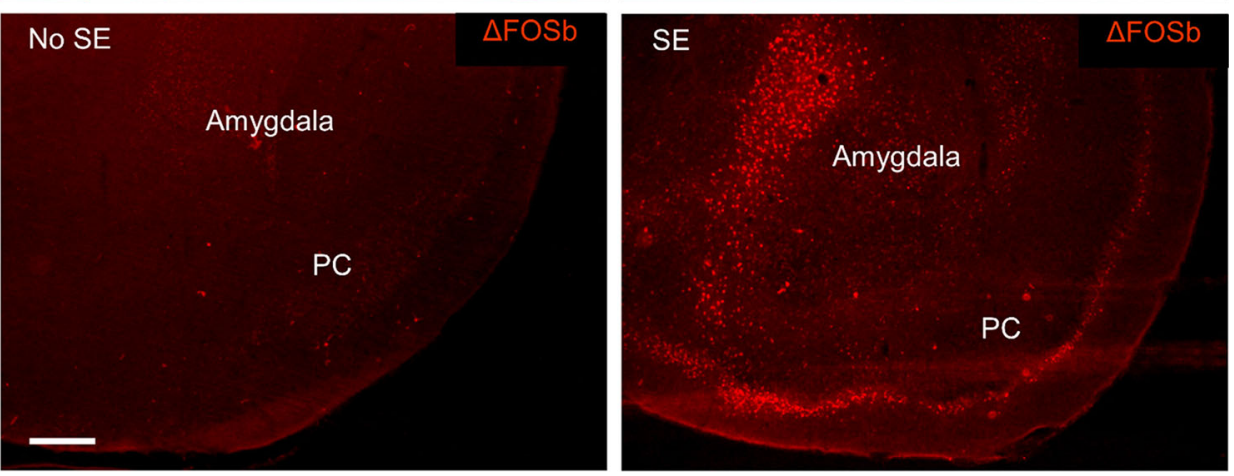

impairment; 2 = severe impairment. All rats were sacrificed at $24 \mathrm{~h}$ or 4 days after SE. Cohorts of rats that experienced SE along with non-seizure controls were anesthetized with isoflurane and sacrificed. The rats were decapitated and their brains were rapidly removed and longitudinally bisected. Hippocampi and cortices were removed from one-half of the brains, rapidly frozen on dry ice and kept for RNA and protein isolation, respectively. The other half was fixed overnight in a $4 \%$ paraformaldehyde solution at $4{ }^{\circ} \mathrm{C}$ and processed for immunohistochemistry and FluoroJade B staining.

\section{Modified Racine Scale for Pilocarpine-Induced SE}

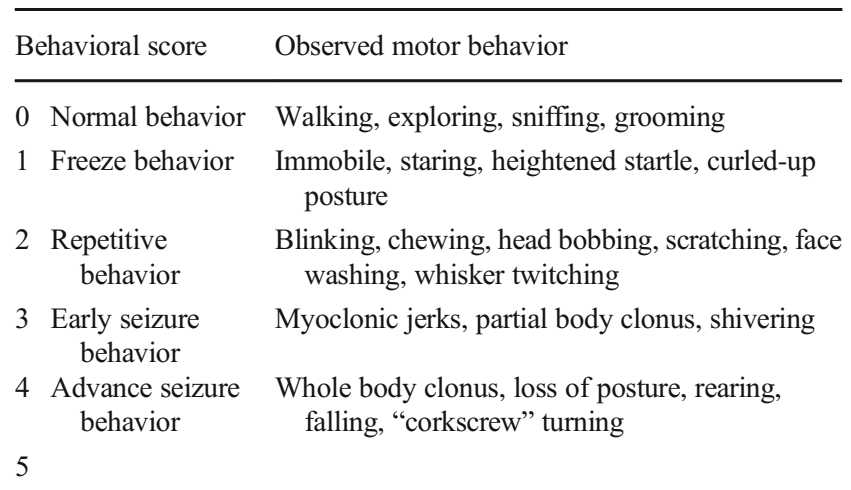

Status epilepticus Repeated seizure activity ( $\geq 2$ events in stages 3,4, (SE)

6 Intense seizure Repetitive jumping or bouncing, wild running, behavior tonic seizures

7 Death

\section{Pharmacokinetics and Administration of TG8-260}

The novel EP2 receptor antagonist TG8-260 was synthesized in our laboratory. Pharmacokinetic analysis and brain distribution of TG8-260 were performed in untreated healthy adult mice at Sai Life Sciences Limited (India). Pharmacokinetics analysis of TG8-260 was previously performed using male mice (data not shown). We repeated these studies with female mice as per the Animal Research: Reporting of In Vivo Experiments (ARRIVE) guidelines by the National Centre of the Replacement, Refinement, and Reduction on Animals in Research (NC3Rs) to determine whether there are sex-related differences in the pharmacokinetics of TG8-260. The data are comparable in both sexes. Female C57BL/6 mice (812 weeks old) weighing between 20 to $35 \mathrm{~g}$ were divided into groups of three. Mice were administered a single intraperitoneal dose of TG8-260 (20 mg/ $\mathrm{kg})$ dissolved in a formulation solution consisting of $5 \% \mathrm{~N}$-methyl pyrrolidone (NMP) and 5\% solutol HS in sterile saline. Blood 
a
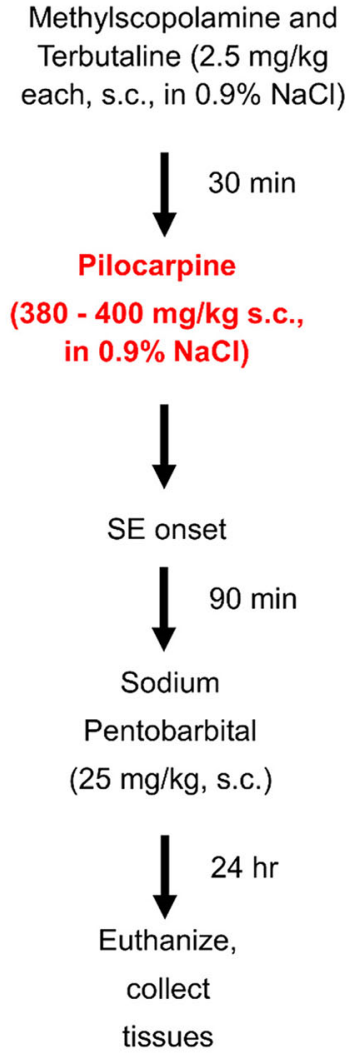

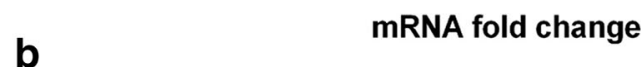

b

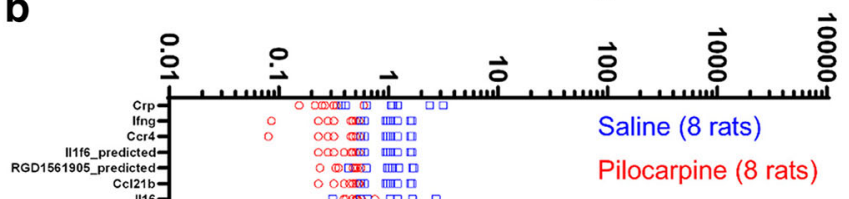

Differentially expressed downregulated

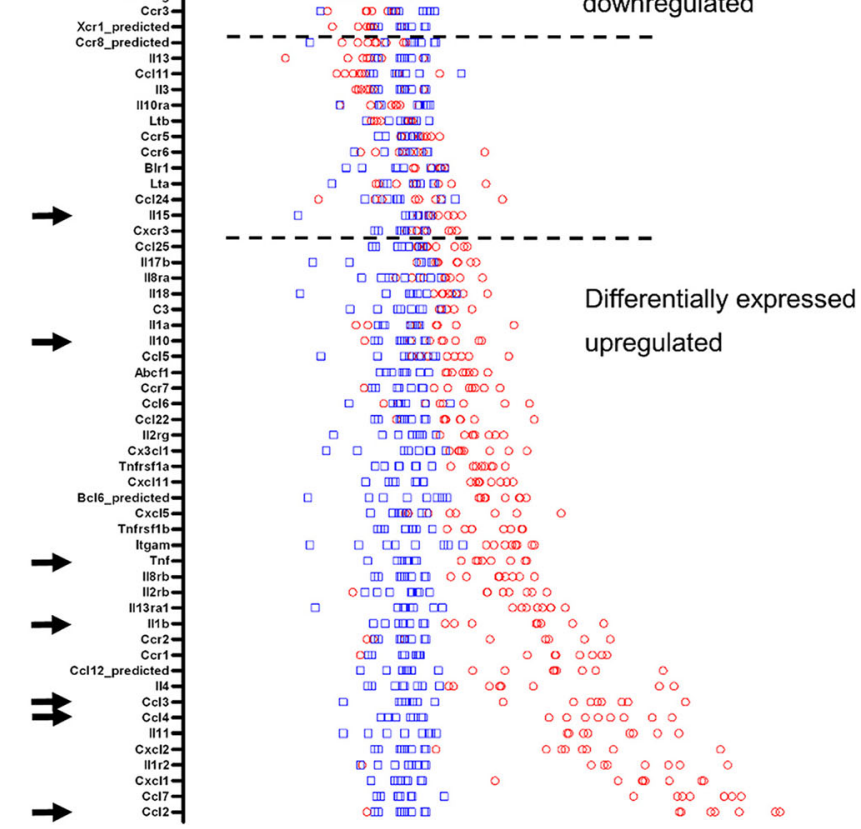

SE following pilocarpine administration (red circles). The genes are arranged on the $y$-axis according to gene expression change after pilocarpine from lowest (top) to highest (bottom). The dashed lines represent the approximate boundaries for differentially expressed downregulated and upregulated mRNAs. Arrows indicate key inflammatory mediators investigated by qRT-PCR 4 days after SE as shown in Fig. 6A. Each symbol represents an individual rat samples (approximately $60 \mu \mathrm{L}$ ) were collected under light isoflurane anesthesia from retro orbital plexus such that samples were obtained at $0.08,0.25,0.5,1,2,4,8$ and $24 \mathrm{~h}$. At each time point, blood samples were collected from three mice. Samples were collected into labeled micro-tubes containing $\mathrm{K}_{2}$ EDTA solution as an anticoagulant. Plasma was immediately harvested from the blood by centrifugation at $4000 \mathrm{rpm}$ for $10 \mathrm{~min}$ at $4 \pm 2{ }^{\circ} \mathrm{C}$ and stored below $-70{ }^{\circ} \mathrm{C}$ until bioanalysis. Immediately after collection of blood, brain samples were collected from cohorts of three mice at each time point. Brain samples were homogenized using ice-cold phosphate buffer saline (pH 7.4) in a ratio of 2:1 (v:w, buffer: brain) and homogenates were stored at $-70 \pm 10{ }^{\circ} \mathrm{C}$ until analysis.

A separate experiment was performed to determine the brain to plasma ratio of TG8-260 in rats. Rats were administered pilocarpine to induce SE. Following $60 \mathrm{~min}$ of SE, the rats were administered diazepam $(10 \mathrm{mg} / \mathrm{kg}$, i.p. $)$ to quell the seizures. One hour after diazepam, the rats received a single intraperitoneal injection of TG8-260 $(25 \mathrm{mg} / \mathrm{kg})$ dissolved in $5 \% \mathrm{~N}$-methyl pyrrolidone (NMP) and 5\% solutol HS in sterile saline. Non-seizure control rats received the same treatment except they were given saline in place of pilocarpine. Blood was collected by cardiac puncture $6 \mathrm{~h}$ after the TG8-260 injection under isoflurane anesthesia. The rats were subsequently perfused with saline to remove the remaining blood and the brain was rapidly removed. The cortex, hippocampus, and striatum were isolated from each brain. TG8-260 was extracted from all samples and sent to Sai Life Sciences for LC-MS/ MS measurements of drug levels.

For the 4 day pilocarpine-induced SE experiments, rats were assigned to a random number stream and received an intraperitoneal injection of TG8-260 $(25 \mathrm{mg} / \mathrm{kg})$ or the vehicle (5\% NMP and 5\% solutol HS in sterile saline) at 2, 8, and $20 \mathrm{~h}$ after status epilepticus onset. The working concentration of TG8-260 was $7.5 \mathrm{mg} / \mathrm{ml}$. Each rat received a volume of either vehicle or TG8-260 based on weight $(3.33 \mathrm{ml} / \mathrm{kg})$. 


\section{FluoroJade B Histochemistry}

The half brains postfixed in $4 \%$ paraformaldehyde were transferred to $30 \%(\mathrm{w} / \mathrm{v})$ sucrose in PBS at $4{ }^{\circ} \mathrm{C}$ the next day until they sank. The brains were then embedded and frozen in optimum cutting temperature (OCT) compound (VWR, West Chester, PA) and subsequently mounted onto a tissue cutting block. Coronal sections $(40 \mu \mathrm{m})$ through the dorsal hippocampus were cut using a cryostat CM 1850 (Leica, Wetzlar, Germany) and placed in $1 \times$ PBS (free-floating). Every sixth section was used for FluoroJade B staining (to label degenerating cells) according to the manufacturer protocol (Histo-chem Inc., Jefferson, AR) as described previously [16]. Briefly, hippocampal sections were mounted on slides and air dried. The slides were then immersed in $100 \%$ ethyl alcohol for $3 \mathrm{~min}$ followed by $1 \mathrm{~min}$ in $70 \%$ alcohol and $1 \mathrm{~min}$ in distilled water. The sections were then transferred to a solution of $0.06 \%$ potassium permanganate for $15 \mathrm{~min}$ and were gently shaken on a rotating platform at $25{ }^{\circ} \mathrm{C}$. The sections were rinsed for $1 \mathrm{~min}$ in distilled water and then transferred to the $0.0004 \%$ FluoroJade staining solution where they were gently agitated in the dark for $30 \mathrm{~min}$. Following staining, the sections were rinsed with three 1-min changes of PBS. The floating sections were mounted onto clean slides and air dried. The sections were made transparent with xylenes and then cover slipped with D.P.X. (Aldrich Chem. Co., Milwaukee, WI) mounting media. FluoroJade labeling was visualized using an Axio Observer A1 epifluorescence microscope equipped with an AxioCam MRc 5 camera and a filter suitable for visualizing fluorescein or FITC (Zeiss, Oberkochen, Germany). Images were acquired using the software AxioVision AC 4.7 (Zeiss).

\section{Quantification of FluoroJade-Labeled Cells}

Following FluoroJade B labeling, images of three hippocampal areas (i.e., hilus, CA1, CA3) were taken under a $\times 5$ objective lens using the AxioVision AC 4.1 software (Zeiss) from each of five sections in each rat. Sections used for FJB quantification were obtained from the dorsal hippocampus between bregma -2.56 and $-4.16 \mathrm{~mm}$ [17] across all rats. A researcher unaware of the experimental conditions counted the number of bright FluoroJade B positive cells in the cell layers. The cell numbers were recorded and expressed as the mean number of positive FluoroJade B (+ FJB) cells/section.

\section{Immunohistochemistry}

Immunohistochemistry was carried out as previously described [4], with minor modifications. Briefly, free-floating sections were washed three times with $1 \times$ PBS for 5 min each and then blocked at $25{ }^{\circ} \mathrm{C}$ for $2 \mathrm{~h}$ with PBS containing $1 \%$ bovine serum albumin (BSA), 10\% normal goat serum
(NGS), and $0.3 \%$ Triton $\mathrm{X}-100$ to reduce nonspecific immunostaining. The sections were then incubated at $4{ }^{\circ} \mathrm{C}$ for $24 \mathrm{~h}$ in the primary antibody (rabbit anti-COX-2, 1:1000; rabbit anti-CCL3, 1:1000, both from Abcam, Cambridge, MA; mouse anti-CD11b, 1:500, Bio-Rad, Hercules, CA; rabbit anti- $\Delta$ FosB, 1:1000, Cell Signaling Technology, Danvers, MA) diluted in antibody dilution solution (ADS), which contains $0.1 \%$ gelatin and $0.3 \%$ Triton X-100 in PBS. After washing three times with ADS, the sections were incubated with Alexa Fluor 488 and 594 conjugated secondary antibodies (Molecular Probes, Grand Island, NY) diluted 1:500 in ADS for $4 \mathrm{~h}$ at $25^{\circ} \mathrm{C}$. The blue-fluorescent Hoechst 33342 dye (Molecular Probes) diluted 1:2000 was also added to the ADS during the secondary antibody incubation. The sections were washed with PBS, attached onto slides, air dried and mounted under FluoroGel mount. Images were taken using an Axio Observer A1 fluorescence microscope and the AxioVision AC 4.7 software. Hippocampal sections in which the primary antibody was omitted showed no staining (data not shown).

For GFAP and Ibal immunohistochemistry, the sections were washed three times with $1 \times$ PBS, blocked for $2 \mathrm{~h}$ in PBS containing $1 \%$ BSA and incubated in the primary antibody [rabbit anti-GFAP, (1:3000), Abcam, Cambridge, MA; rabbit anti-Ibal (1:3000), Wako, Richmond, VA] diluted in ADS at $4{ }^{\circ} \mathrm{C}$ for $24 \mathrm{~h}$. After washing three times with ADS, the sections were incubated with Alexa Fluor goat anti-rabbit 594 secondary antibody (1:2000) for GFAP; Alexa Fluor goat anti-rabbit 488 (1:2000) for Iba1 (Molecular Probes, Eugene, OR) diluted in ADS for $4 \mathrm{~h}$ at $25^{\circ} \mathrm{C}$. The sections were again washed with PBS, air dried, and mounted under a coverslip with FluoroGel mounting media. Images were taken using an Axio Observer A1 fluorescence microscope and the AxioVision AC 4.1 software. Sections treated in a similar manner except that the primary antibodies were omitted showed no staining. All of the sections used for immunohistochemistry were obtained from the dorsal hippocampus between bregma -2.56 and $-4.16 \mathrm{~mm}$ [17] across all rats.

\section{Rat Inflammatory Cytokines and Receptors PCR Array}

The rat inflammatory cytokines and receptors PCR array (SABiosciences) was used to detect changes in expression of 84 inflammatory cytokine transcripts according to the manufacturer's protocol. Total RNA was isolated as described [7]. First strand cDNA was generated using $1 \mu \mathrm{g}$ of total RNA from each sample and the SABiosciences $\mathrm{RT}^{2}$ first strand kit (SABiosciences, Frederick, MD) according to the manufacturer's protocol. The first strand cDNA made from RNA described above was added to a mixture containing SYBR green. This cocktail was added to a 96-well PCR plate preloaded with primers for 84 inflammatory cytokine transcripts. The real-time PCR reaction was carried out for 40 cycles using 
a Bio-Rad IQ5 optical system. RNA expression of cytokines was normalized using the average of five housekeeping genes (ribosomal protein lateral stalk subunit P1, Rplp1; hypoxanthine phosphoribosyl transferase 1, Hprt; ribosomal protein L13a, Rpl13a; lactate dehydrogenase A, Ldha; beta actin, Actb) included in the array.

\section{Quantitative PCR}

The qPCR was performed as previously described with minor modifications [4, 8]. Melting curve analysis was used to verify specificity of the primers by single-species PCR product (Table 1). The cDNA was more diluted in these experiments than previously reported $[4,8]$ resulting in higher but detectable CT values. Three housekeeping genes ( $\beta$-actin, glyceraldehyde-3-phosphate (GAPDH), and hypoxanthine phosphoribosyl transferase 1 (HPRT1)) (Table 2) were used as internal controls. Samples without cDNA template served as the negative controls. The 10 inflammatory mediators investigated are listed with the primer sequences in Table 1. Interleukin 1-beta (IL-1 $\beta$ ), interleukin 6 (IL-6), interleukin 15 (IL-15) and tumor necrosis factor alpha (TNF $\alpha)$ are produced and secreted by cells involved in both innate and acquired immunity to stimulate inflammation. C-C motif chemokine ligands 2, 3, and 4 (CCL2, CCL3, and CCL4) as well as $\mathrm{C}-\mathrm{X}-\mathrm{C}$ Motif Chemokine Ligand 10 (CXCL10) are chemokines that recruit peripheral leukocytes to sites of inflammation or injury, although CXCL10 can also exert direct excitatory effects on neurons [18]. Also investigated is interleukin 10 (IL-10) whose delayed induction acts to resolve inflammation in a murine model of wound healing [19].
Cyclooxygenase 2 (COX-2) is an intracellular rate-limiting enzyme in the production of prostanoids that contribute to the inflammatory response. Analysis of quantitative realtime PCR data for each gene of interest in each sample was performed by subtracting the geometric mean of the three internal housekeeping genes from the measured cycle threshold value obtained from the log phase of the amplification curve to yield $\Delta \mathrm{CT}$ for relative quantification. The fold change of each gene of interest was estimated for each animal 4 days after pilocarpine-induced SE relative to the amount of RNA found in the control animals using the $2^{-\Delta \Delta C T}$ method [20]. All conditions for qRT-PCR were the same. The $\Delta \Delta \mathrm{CT}$ values were used for statistical comparison of qRT-PCR.

\section{Western Blot}

Cortical lysates were used for western blot. The supernates (30 $\mu \mathrm{g}$ of protein each) were resolved by $10 \%$ sodium dodecyl sulfate polyacrylamide gel electrophoresis (SDS-PAGE) and electroblotted onto PVDF membranes (Millipore). Membranes were blocked with $5 \%(\mathrm{w} / \mathrm{v})$ non-fat milk at $25{ }^{\circ} \mathrm{C}$ for $4 \mathrm{~h}$ and then incubated overnight at $4{ }^{\circ} \mathrm{C}$ with the primary antibody: rabbit anti-albumin (1:2000, Abcam), rabbit anti-COX-2 (1:2000, Abcam) or mouse anti- $\beta$-Actin (1:1000, Abcam). This procedure was followed by incubation with horseradish peroxidase-conjugated secondary antibodies (1:2000, Jackson Immuno) at room temperature for $2 \mathrm{~h}$. The blots were developed by enhanced chemiluminescence (ECL) (Thermo Fisher Scientific) and scanned using a ChemiDoc MP imaging system (Bio-Rad, Hercules, CA). The band intensities were quantified by ImageLab 6.0.1 (Bio-Rad).

Table 1 Real-time rat PCR primer sequences. The approved human gene nomenclature symbol is in parentheses if different from gene name

\begin{tabular}{lll}
\hline Genes: & Forward primer (sequence 5'-3'): & Reverse primer (sequence 5'-3'): \\
\hline HPRT1 & GGTCCATTCCTATGACTGTAGATTTT & CAATCAAGACGTTCTTTCCAGTT \\
$\beta$-ACTIN (ACTB) & CCAACCGTGAAAAGATGACC & ACCAGAGGCATACAGGGACA \\
GAPDH & GGTGAAGGTCGGTGTGAAC & CCTTGACTGTGCCGTTGAA \\
CCL2 & CAGAAACCAGCCAACTCTCA & GTGGGGCATTAACTGCATCT \\
CCL3 & TCCACGAAAATTCATTGCTG & AGATCTGCCGGTTTCTCTTG \\
CCL4 & CATCGGAACTTTGTGATGGA & CACAGATTTGCCTGCCTTTT \\
IL-1 $\beta$ (IL1B) & CAGGAAGGCAGTGTCACTCA & TCCCACGAGTCACAGAGGA \\
IL-6 (IL6) & AACTCCATCTGCCCTTCAGGAACA & AAGGCAGTGGCTGTCAACAACATC \\
TNF $\alpha$ (TNF) & CGTAGCCCACGTCGTAGC & GGTTGTCTTTGAGATCCATGC \\
COX-2 (PTGS2) & ACCAACGCTGCCACAACT & GGTTGGAACAGCAAGGATTT \\
IL-10 (IL10) & ACAGATTCCTTACTGCAGGACTTT & CAAATGCTCCTTGATTTCTGG \\
IL-15 (IL15) & CGATCTGGAGAAAATTGAAAGTC & CTGTACTCGTGCAAAATAACCTGT \\
GFAP 10 & GTGCTGCTGAGTCTGAGTGG & TTGCAGGAATGATTTCAAGTTTT \\
lba1 (AIF1) & CATCTCCACCGTCTTTACCAC & AACCGCATCACCATTCCTG \\
CD11B (ITGAM) & TCGATATCTCCATTGCCATTCAG & GATGGGATCAAACAAGCACTTC \\
\hline
\end{tabular}


Table 2 CT values and geometric means of housekeeping genes in four groups of rats for 4-day inflammatory mediator measurement

\begin{tabular}{|c|c|c|c|c|}
\hline Genes: & Saline vehicle (10 rats) & Saline TG6-10-1 (8 rats) & Pilocarpine vehicle (9 rats) & Pilocarpine TG6-10-1 (9 rats) \\
\hline HPRT1 & $26.7 \pm 0.5$ & $28.5 \pm 0.9$ & $29.9 \pm 1.2$ & $27.0 \pm 0.6$ \\
\hline$\beta$-ACTIN & $27.4 \pm 0.6$ & $29.6 \pm 0.9$ & $29.8 \pm 1.4$ & $27.2 \pm 0.9$ \\
\hline GAPDH & $26.3 \pm 0.6$ & $28.7 \pm 0.9$ & $29.8 \pm 1.4$ & $26.5 \pm 0.6$ \\
\hline Geomean & $26.8 \pm 0.6$ & $28.9 \pm 0.9$ & $29.8 \pm 1.3$ & $26.9 \pm 0.6$ \\
\hline
\end{tabular}

Data are the mean \pm standard error

\section{Cell Culture}

BV2-hEP2 cells (murine BV2 cells expressing the human EP2 receptor) were created in our laboratory and cultured as previously described [21]. The cells were grown and maintained in media containing: Dulbecco's modified Eagle's medium (DMEM)/F12 (Invitrogen, Carlsbad, CA), supplemented with $10 \%$ (v/v) fetal bovine serum (Invitrogen), 100 units $/ \mathrm{mL}$ penicillin, $100 \mu \mathrm{g} / \mathrm{mL}$ streptomycin (Invitrogen), and $800 \mu \mathrm{g} /$ $\mathrm{mL}$ G418 (Sigma). The rat C6 glioma (C6G) cells stably expressing human DP1, EP2, EP4, or IP receptors were created in our laboratory $[1,22,23]$ and grown in Dulbecco's Modified Eagle Medium (DMEM) (Invitrogen) supplemented with $10 \%(\mathrm{v} / \mathrm{v})$ fetal bovine serum (FBS) (Invitrogen), $100 \mathrm{U} /$ $\mathrm{ml}$ penicillin, $100 \mu \mathrm{g} / \mathrm{ml}$ streptomycin (Invitrogen), and $600 \mu \mathrm{g} / \mathrm{ml} \mathrm{G} 418$ (Invitrogen). All cell cultures were incubated at $37{ }^{\circ} \mathrm{C}$ in $95 \%$ air / $5 \% \mathrm{CO}_{2}$, and the culture medium was replaced every 3-4 days with fresh medium.

\section{Cell-Based cAMP Assay}

Intracellular cAMP was measured with a cell-based homogeneous time-resolved fluorescence resonance energy transfer (TR-FRET) method (Cisbio Bioassays), as previously described [1]. The assay is based on generation of a strong FRET signal upon the interaction of two molecules, an anticAMP antibody coupled to a FRET donor (Cryptate) and cAMP coupled to a FRET acceptor (d2). Endogenous cAMP produced by cells competes with labeled cAMP for binding to the cAMP antibody and thus reduces the FRET signal. Cells stably expressing human DP1, EP2, EP4, or IP receptors were seeded into 384-well plates in $40 \mu \mathrm{l}$ complete medium (4000 cells/well) and incubated overnight. The medium was carefully withdrawn and $10 \mu$ l Hanks' Buffered Salt Solution (HBSS) (Hyclone) containing $20 \mu \mathrm{M}$ rolipram was added into the wells to block phosphodiesterases. The cells were incubated at room temperature for $0.5-1 \mathrm{~h}$ and then treated with vehicle or test compound for $10 \mathrm{~min}$ before addition of increasing concentrations of appropriate agonists: BW245C for DP1, $\mathrm{PGE}_{2}$ for EP2 and EP4, or iloprost for IP. The cells were incubated at room temperature for $40 \mathrm{~min}$, then lysed in $10 \mu l$ lysis buffer containing the FRET acceptor cAMP-d2 and 1 min later another $10 \mu l$ lysis buffer with anti-cAMP-
Cryptate was added. After 60-90 min incubation at room temperature, the FRET signal was measured by an Envision 2103 Multilabel Plate Reader (PerkinElmer Life Sciences) with a laser excitation at $337 \mathrm{~nm}$ and dual emissions at $665 \mathrm{~nm}$ and $590 \mathrm{~nm}$ for $\mathrm{d} 2$ and Cryptate (50- $\mu$ s delay), respectively. The FRET signal was expressed as: F665/F590 $\times 10^{4}$.

\section{Anti-inflammatory Assay in BV2-hEP2 Cells}

Stable BV2-hEP2 microglia cells [21] were incubated overnight on poly-D-lysine coated 12 well plates at 200,000 cells/ well in culture media. The cells were treated with vehicle or either 0.3 or $1 \mu \mathrm{M}$ TG8-260 for $1 \mathrm{~h}$, followed by $30 \mathrm{nM}$ of the EP2 agonist ONO-AE1-259-01 or its vehicle for $1 \mathrm{~h}$, and then $100 \mathrm{ng} / \mathrm{mL}$ lipopolysaccharide (LPS, Sigma, USA) or its vehicle for $2 \mathrm{~h}$. For vehicle treatment, a similar dilution of the compound vehicle (DMSO) in complete media was used. All compounds were dissolved in DMSO and diluted in media just prior to cell treatment. After all treatments, the entire media was removed from all of the wells, and RNAs were extracted from the treated cells using an RNA extraction kit (Zymo Research, USA) as recommended by the manufacturer's protocol. RNA samples were converted into cDNA using a cDNA conversion kit (Quanta, USA) and further used for qPCR analysis. SYBR Green super mix (Quanta, USA) and primers for glyceraldehyde-3-phosphate dehydrogenase (GAPDH), IL-10, CCL2, CCL3 and CCL4 genes (IDT, USA, see Table 3 for the primer sequences) were used for the PCR reaction carried out in a CFX96 Real-Time System (Bio-Rad). PCR data were presented as mean fold changes of gene expression in all of the treated samples normalized to vehicle-treated cells. GAPDH was used as a single internal control for relative quantification.

\section{Chemicals and Drugs}

$\mathrm{PGE}_{2}$, butaprost, BW245C, and iloprost were purchased from Cayman Chemicals. Methylscopolamine, terbutaline, pilocarpine- $\mathrm{HCl}$, rolipram and lipopolysaccharide were purchased from Sigma-Aldrich. ONO-AE1-259-1 was generously provided by ONO Pharmaceuticals (Osaka, Japan). The EP2 receptor antagonist TG8-260 was developed and synthesized in our laboratory during the lead optimization of the scaffold 
Table 3 Real-time mouse PCR primer sequences for BV2-hEP2 experiments. The approved human gene nomenclature symbol is in parentheses if different from gene name

\begin{tabular}{lll}
\hline Genes: & Forward primer (sequence 5'-3'): & Reverse primer (sequence 5'-3'): \\
\hline GAPDH & TGTCCGTCGTGGATCTGAC & CCTGCTTCACCACCTTCTTG \\
IL-10 (IL10) & ATTTGAATTCCCTGGGTGAGAAG & CACAGGGGAGAAATCGATGACA \\
CCL2 & CATCCACGTGTTGGCTCA & GCTGCTGGTGATCCTCTTGTA \\
CCL3 & TGCCCTTGCTGTTCTTCTCT & GTGGAATCTTCCGGCTGTAG \\
CCL4 & CATGAAGCTCTGCGTGTCTG & GGAGGGTCAGAGCCCATT \\
\hline
\end{tabular}

(Ganesh et al., 2018; Amaradhi et al. 2020). The structure of TG8-260 compound was characterized by nuclear magnetic resonance (NMR), liquid chromatography mass spectrometry (LC/MS) and purity was determined to $>97 \%$ by highperformance liquid chromatography (HPLC).

\section{Data Analysis}

Data are presented as individual points with the means and 95\% confidence interval shown. Statistical analysis was performed with GraphPad Prism version 7 (GraphPad software, San Diego, CA). Student's $t$ test, Mann-Whitney test or ANOVA were performed as appropriate based on the questions asked and the comparisons made to examine differences of chemical effects. Student's $t$ test was used for comparisons made with parametric data and the Mann-Whitney test for comparisons made with nonparametric data for only two group data sets. ANOVA was used for comparisons made with parametric data of three or more groups. The differences were considered to be statistically significant if $p<.05$. The Shapiro-Wilk test in Origin 9.4.2 (OriginLab, Northampton, MA) was used to test normality of the data. Power analysis was performed with $G^{*}$ Power3.1.9.2 (Universitat Kiel, Germany). Estimation statistics was performed using an internet application (http://www.estimationstats.com/\#/) built for common use by Dr. Adam Claridge-Chang and Mr. Joses Ho. The application performs data analysis with a bootstrap estimation (DABEST) package in python [24] with 5000 resampling, bias-corrected (to correct for bias and skewness in the distribution of the bootstrap estimates), and accelerated bootstrap analysis to determine the confidence interval of differences between treatment groups. The mean differences between groups and the confidence interval from the bootstrapping are shown.

\section{Results}

\section{Pilocarpine-Induced Neuropathology}

Experiments were performed using a rat pilocarpine SE model to investigate the importance of the EP2 receptor in seizure- induced neuropathology. Adult male Sprague-Dawley rats were treated with pilocarpine according to the protocol in Fig. 1A. Methylscopolamine and terbutaline are supporting agents that do not alter seizure characteristics and are given to improve survival of rats administered such a high dose of pilocarpine. Rats were administered diazepam to interrupt SE according to the dosing paradigm in Fig. 1A.

In rats, seizure-induced behaviors began within minutes of pilocarpine administration and consisted of distinct motor behaviors that include tail extension, forelimb clonus and whole body clonic seizures. These behaviors were observed and scored using a modified Racine scale (see methods). Fluorescence immunohistochemistry performed on coronal hippocampal sections $(40 \mu \mathrm{m})$ revealed robust induction of $\Delta$ Fosb in neurons in seizure sensitive limbic areas (hippocampus, amygdala and piriform cortex) $24 \mathrm{~h}$ after SE onset (Fig. $1 \mathrm{~B}, \mathrm{C})$, confirming increased neuronal activity in limbic regions of the brain.

\section{Expression Pattern of Inflammatory Mediators $24 \mathrm{~h}$ after SE}

We previously showed that pilocarpine-induced SE changes the expression of a small number of inflammatory cytokines and their receptors during and after SE in mice [1-3, 9, 25]. To gain insight into how broad the neuroinflammation is in the hippocampus $24 \mathrm{~h}$ after pilocarpine-induced SE (Fig. 2A), we investigated the expression profile of a panel of inflammatory mediators and receptors using the PCR array technology offered by SA Biosciences. The RT-profiler was used to measure RNA expression of 84 inflammatory mediators in the hippocampus. RNA was isolated from the hippocampus of two cohorts of rats [saline-treated non-seizure controls $(n=8$ rats) and rats administered pilocarpine that experienced SE ( $n=8$ rats)]. Of the 84 mRNAs investigated, 21 were not included in the analysis due to high variation observed within the saline-treated non-seizure control group with outliers identified by Grubb's test. A threshold of a 2-fold change (up or down) relative to saline was used to identify differentially expressed genes after pilocarpine treatment. Of the 63 genes included in the analysis, 50 were differentially expressed (13 downregulated and 37 upregulated (Fig. 2B). These data 
reveal a robust seizure-induced change of inflammationrelated transcripts in the hippocampus $24 \mathrm{~h}$ after pilocarpineinduced SE.

\section{Potency and Selectivity of the Novel EP2 Antagonist TG8-260}

A continued medicinal chemistry and lead optimization campaign to improve solubility and selectivity of our firstgeneration small-molecule EP2 antagonists led to the identification of a second-generation EP2 receptor antagonist, TG8260. Structurally, TG8-260 does not have an acrylamide moiety like the earlier lead EP2 antagonist, TG6-10-1, and displays good aqueous solubility $(238 \mu \mathrm{M}$ in $\mathrm{pH} 7.4 \mathrm{PBS}$ with $1 \%$ DMSO). Using a cAMP-mediated TR-FRET-based functional EP2 assay [1], we found that TG8-260 inhibits $\mathrm{PGE}_{2}-$ induced EP2 receptor activation in a concentration-dependent manner in a C6-glioma cell line overexpressing human EP2 receptors. In this assay, TG8-260 displayed a competitive mechanism of antagonism of EP2 receptors, with a Schild $K_{\mathrm{B}}$ of $13.2 \mathrm{nM}$ (mean of 3 independent experiments). TG8260 displayed a $>500$-fold selectivity to EP2 versus other $G_{\text {as }}$-coupled receptors such as DP1, EP4 and IP, which yielded $K_{\mathrm{B}}$ values of 8,45 , and $10 \mu \mathrm{M}$, respectively. The relatively high solubility of TG8-260 allowed for the use of a more gentle formulation strategy in animal experiments described in the subsequent sections.

\section{In Vitro Modulation of Inflammation by TG8-260}

Neuroinflammation has emerged as an important feature of acute brain injuries and chronic neurodegenerative diseases $[26,27]$. We demonstrated a robust induction of a panel of inflammatory mediators in the hippocampus of rats following pilocarpine-induced SE shown in Fig. 2. We next investigated the inflammation modulating properties of TG8-260 in vitro. Using BV2-hEP2 cells [21], we determined whether inhibition of the EP2 receptor by TG8-260 displays altered inflammatory actions on a small subset of inflammatory mediators. Upon activation of BV2-hEP2 cells with $100 \mathrm{ng} / \mathrm{mL}$ LPS, mRNA levels of three chemokines (CCL2, CCL3 and CCL4), but not IL-10 were induced within $2 \mathrm{~h}$ (Fig. 3). The specific EP2 agonist ONO-AE1-259-01 at $30 \mathrm{nM}$ reduced the induction of CCL2, CCL3 and CCL4 by LPS. Treatment with TG8-260 $(0.3$ and $1 \mu \mathrm{M})$ significantly antagonized the effects of the combination of LPS and ONO-AE1-259-1 on IL-10, CCL3 and CCL4 (Fig. 3). Although a trend for antagonism by TG8-260 was observed for CCL2, significance was not attained due to higher variation. In a control experiment, incubation of the BV2-hEP2 cell line with TG8-260 $(1 \mu \mathrm{M})$ in the presence or absence of LPS did not show a significant effect on the induction of inflammatory mediators (not shown). These data support the use of TG8-260 as an inflammatory modulator in in vivo disease models that involve COX-2 induction.

\section{Pharmacokinetics of TG8-260}

A single injection of TG8-260 (20 mg/kg, i.p.) in adult female C57BL/6 mice revealed a terminal plasma half-life $\left(\mathrm{T}_{1 / 2}\right)$ of $2.8 \mathrm{~h}$ and a relatively low brain concentration observed only up to $4 \mathrm{~h}$, with a brain to plasma ratio 0.02 (Fig. $4 \mathrm{~A}$ ). In rats, a single injection of TG8-260 (25 mg/kg, i.p.) achieved a 2-fold higher concentration in the brain following pilocarpineinduced SE in comparison to saline injected non-SE control rats $(146 \pm 32 \mathrm{ng} / \mathrm{g}$ for SE rats, $(n=7$ rats $)$ versus $75 \pm 15 \mathrm{ng} / \mathrm{g}$ for control rats $(n=6$ rats)) (Fig. 4B). A brain to plasma concentration ratio of $0.03 \pm 0.004$ was calculated in salinetreated rats $6 \mathrm{~h}$ after a single intraperitoneal injection of TG8-260 $(25 \mathrm{mg} / \mathrm{kg})$. The average brain to plasma ratio of TG8-260 was significantly higher $(0.05 \pm 0.007, p=.04, t$ test) in rats that experienced pilocarpine-induced SE and were administered TG8-260 $6 \mathrm{~h}$ earlier. This is consistent with opening of the BBB after SE.

\section{TG8-260 Reduces Hippocampal Inflammation}

In total, 50 adult male Sprague-Dawley rats were used to compare the effect of TG8-260 on neuropathology caused by SE. Twenty rats were included as non-seizure controls and were administered saline instead of pilocarpine. Pilocarpine was administered to the other 30 rats according to the dosing paradigm in Fig. 5A, all of which entered SE resulting in a $100 \%$ success rate. Unfortunately, 13 of 30 rats died from a tonic seizure during the ensuing SE resulting in a $43 \%$ total mortality.

Neuroinflammation observed in the days following SE in rats is characterized by the transient upregulation of inflammatory mediators including cytokines (interleukin 1 beta, IL- $1 \beta$; tumor necrosis factor alpha, $\mathrm{TNF} \alpha$; interleukin 6, IL-6), chemokines (chemokine (C-C motif) ligand 2, CCL2; CCL3; CCL4; C-X-C motif chemokine 10, CXCL10) and proinflammatory enzymes [cyclooxygenase-2 (COX-2) and NADPH oxidase 2 (NOX2)]. qRTPCR was carried out to measure the expression of ten inflammatory mediators, a majority of which were previously shown to be upregulated in the rat brain following SE induced by diisopropylfluorophosphate $[4,7,8]$ and $24 \mathrm{~h}$ after pilocarpine-induced SE (Fig. 2). Four days after pilocarpine-induced SE, rats administered three doses of vehicle showed increased hippocampal mRNA levels in all ten of the inflammatory mediators (Fig. 5B). However, rats administered three doses of EP2 antagonist TG8-260 following pilocarpine-induced SE displayed a broadly blunted inflammatory response (Fig. 5B). The seizure-induced upregulation of these mRNAs was 

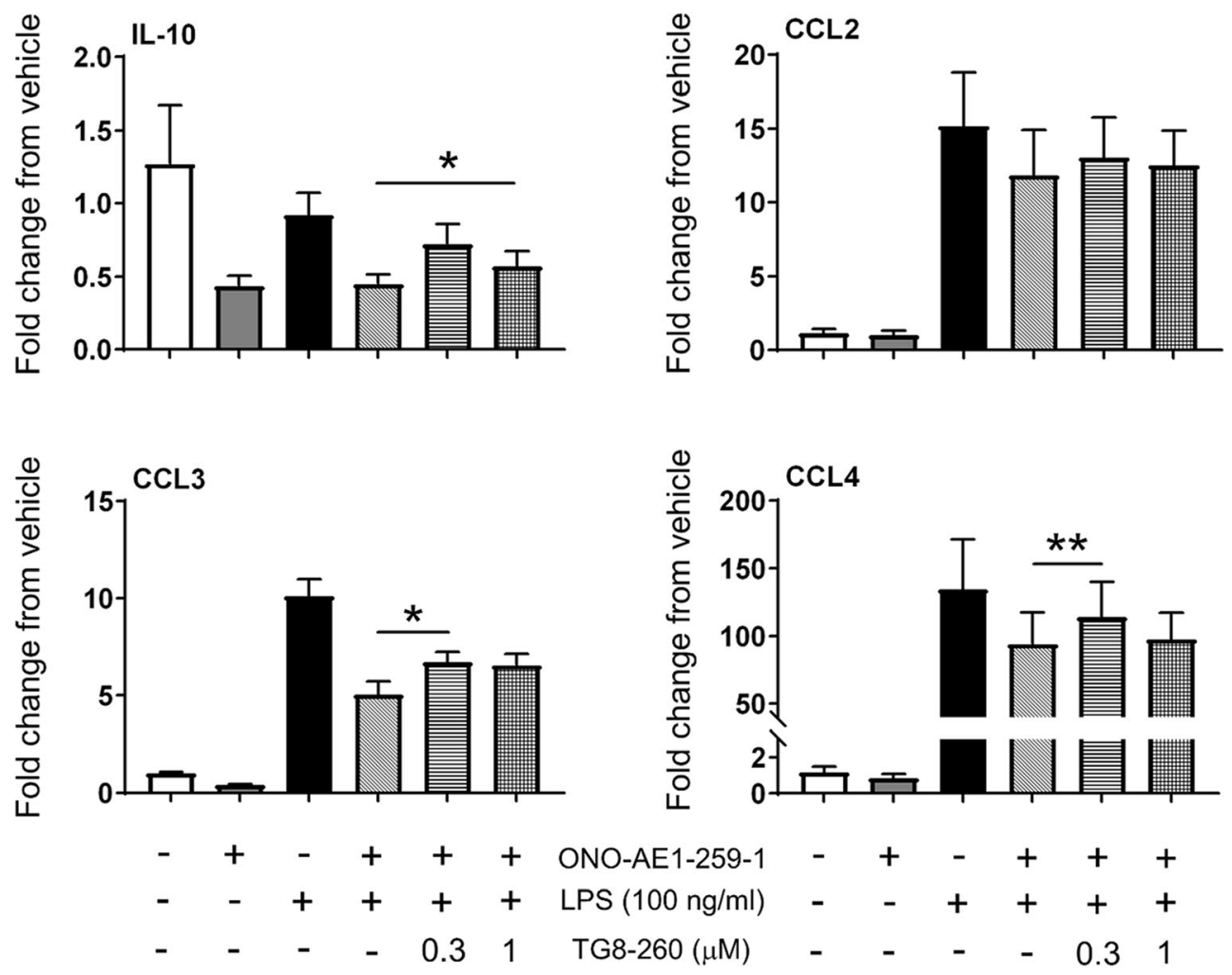

Fig. 3 TG8-260 modulates LPS induced inflammation in the BV2-hEP2 cell line. Shown are the mean fold change in mRNA expression of a small panel of inflammatory mediators in BV2-hEP2 cells upon treatment with lipopolysaccharide (LPS, $100 \mathrm{ng} / \mathrm{ml}$ ), the EP2 agonist ONO-AE1-259-01 $(30 \mathrm{nM})$ and TG8-260 (0.3 or $1 \mu \mathrm{M})$. BV2-hEP2 were seeded at 200,000 cells/well and incubated overnight. The cultures were treated in duplicate with vehicle, TG8-260 or ONO-AE1-259-01 for $1 \mathrm{~h}$ and then LPS or its vehicle were added for an additional $2 \mathrm{~h}$. Analyte mRNAs were measured

reduced by $\sim 72 \% 4$ days after pilocarpine-induced SE (40 \pm 17 average fold induction for vehicle vs $11 \pm 4$ average fold induction for TG8-260, $p=.00002$, paired $t$ test on $\Delta \Delta \mathrm{CT}$ values).

Of the 10 inflammatory mediators tested CCL3 mRNA displayed the highest fold induction in rats administered the vehicle as measured 4 days after pilocarpine-induced SE (Fig. 5B). CCL3 was also one of the highest 10 induced transcripts following pilocarpine-induced SE measured $24 \mathrm{~h}$ after SE in the 84 transcript RT-PCR experiment (Fig. 2). CCL3 mRNA was significantly lower in rats that were injected with three doses of TG8-260 after pilocarpine-induced SE [107 \pm 33 average fold induction for vehicle ( $n=9$ rats) versus $26 \pm 8$ average fold induction for TG8-260 ( $n=9$ rats); $p=.01$, $t$ test using $\Delta \Delta \mathrm{CT}$ values (Fig. 5C). Fluorescence immunohistochemistry performed on $40 \mu \mathrm{m}$ coronal hippocampal sections revealed CCL3 upregulation in microglia located in the hippocampus and amygdala (Fig. 6) $24 \mathrm{~h}$ following pilocarpineinduced SE compared to non-seizure control rats. Similar, intense CCL3 immunostaining in CD11b positive microglia was also detected in the piriform cortex and thalamus in rats that experienced SE $24 \mathrm{~h}$ earlier (not shown). by qRT-PCR (see Table 3 for primer sequences). Although fold changes are shown in the figure, $\Delta \Delta \mathrm{CT}$ values were used for statistical analysis as they are normally distributed. Differences in chemical treatment were analyzed by repeated measures ANOVA-with post hoc Holm-Sidak multiple comparisons test. $*=p<.05, * *=p<.01$. The experiment was repeated with five independent cultures of different passages. Data are mean \pm standard error of the mean

Another key inflammatory mediator robustly induced following pilocarpine-induced SE is the rate-limiting enzyme in the production of prostaglandins, $\mathrm{COX}-2$ [3, 4, 9]. Fluorescence immunohistochemistry performed on coronal hippocampal sections $(40 \mu \mathrm{m})$ revealed COX-2 upregulation in the hippocampus, amygdala, and parietal cortex 4 days following pilocarpine-induced SE compared to rats that did not experience SE (Fig. 7A, B). The induction of COX-2 appeared to be in neurons in these seizure sensitive brain nuclei (cf Fig. 7A, B). Western blot was carried out to quantify the level of COX-2 induction following pilocarpine-induced SE. The level of COX-2 protein on day 4 in cortical homogenates was significantly higher in rats administered vehicle after SE ( $n=5$ rats) compared to rats administered TG8-260 $(n=5$ rats) $(7 \pm 1.9$ fold increase relative to $\beta$-actin for vehicle versus $2 \pm 0.2$ fold increase relative to $\beta$-actin for TG8-260, $p=.03, t$ test) (Fig. 7C, D). Taken together, these data show that exposure of rats to pilocarpine and subsequent $\mathrm{SE}$ induces an inflammatory cascade involving COX-2 and several cytokines and chemokines including CCL3; however, TG8-260 administration after $\mathrm{SE}$ attenuates the inflammatory response normally observed 4 days later. 


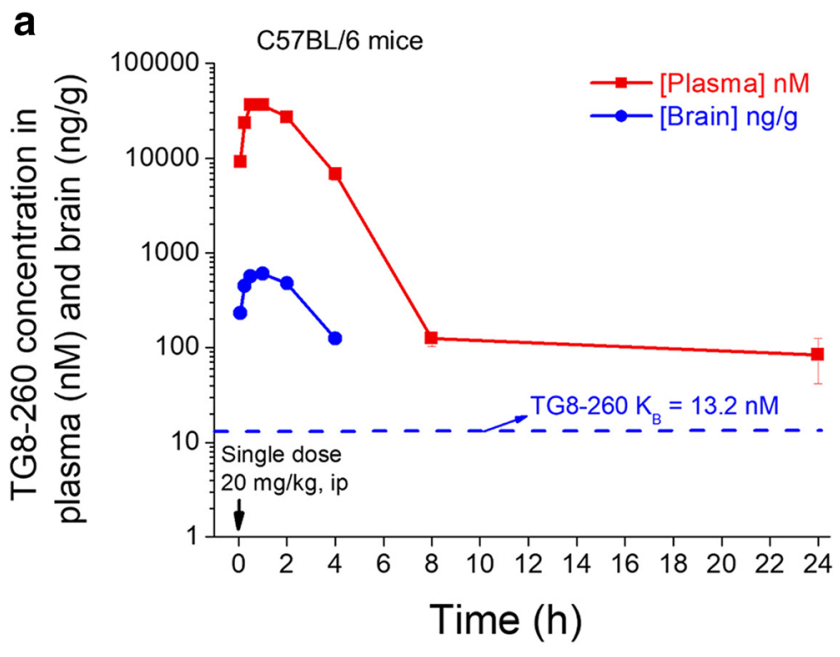

b

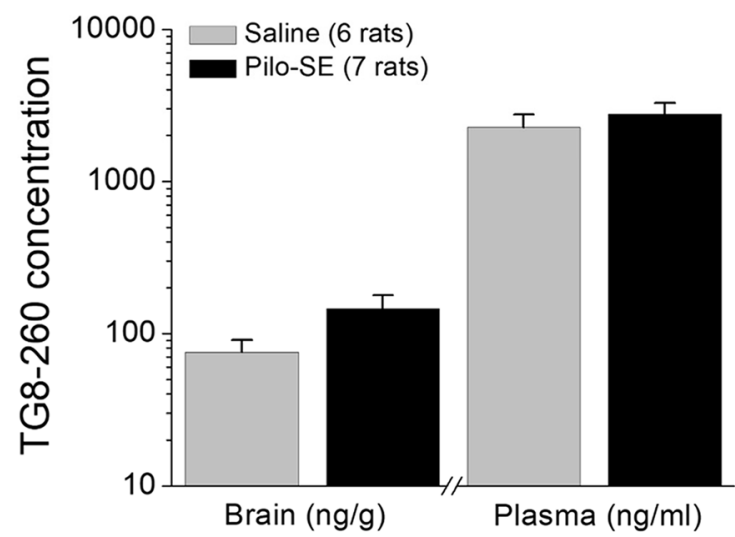

Fig. 4 Pharmacokinetics of TG8-260. (A) the plasma and brain levels of TG8-260 in adult female C57BL/6 mice that received TG8-260 in the vehicle $5 \%$ NMP, $5 \%$ solutol-HS15, 90\% saline (mean \pm standard error of the mean, $n=3$ mice) (single dose, $20 \mathrm{mg} / \mathrm{kg}$, i.p.). More than a 7 -fold plasma concentration above the $K_{\mathrm{B}}$ value was attained at $24 \mathrm{~h}$. (B) brain and plasma levels of TG8-260 in saline and pilocarpine-induced SE rats. TG8-260 (25 mg/kg, i.p.) dissolved in the vehicle 5\% NMP, 5\% solutolHS15, 90\% saline was administered $1 \mathrm{~h}$ after saline or termination of pilocarpine-induced SE by diazepam, and whole blood and brain was collected 6 h later. $n=6$ rats for saline and 7 rats for pilocarpine-SE. Data are mean \pm standard error of the mean. The amount of TG8-260 present in the samples was quantified by LC-MS/MS analysis. The concentration of TG8-260 in the brain of SE rats is 2-fold higher than control rats

\section{TG8-260 Attenuates Hippocampal Gliosis}

Gliosis is defined as the morphological and metabolic reaction of glial cells to injury. Astrogliosis (astrocyte activation) and microgliosis (microglia activation) after SE can be revealed by an increase in the size of the cell body following positive immunohistochemical staining of astrocytic glial fibrillary acidic protein (GFAP) and microglial ionized calciumbinding adapter molecule 1 (Iba1). Alternatively, quantification of mRNA levels of these two glial markers is often associated with gliosis that occurs following SE in rodents. Experiments were performed to determine the features of glial activation after pilocarpine-induced SE. There appeared to be an increase in the size of the cell body of microglia but not astrocytes in rats that experienced SE and were administered the vehicle compared to rats injected with TG8-260 after SE (Fig. 8A-D). The microglia displayed a more amoeboid appearance in rats administered the vehicle after pilocarpineinduced SE compared to rats injected with TG8-260. The astrocytes displayed a very similar morphology in rats administered TG8-260 compared to the vehicle. Additionally, mRNA of astrocytic GFAP, microglial Iba1 and CD11b was measured in the hippocampus of rats after SE to quantify gliosis. Compared to saline controls, rats that experienced SE 4 days earlier and were administered the vehicle showed elevated GFAP, Iba1 and CD11b mRNA (Fig. 8E). Rats administered three doses of TG8-260 after pilocarpine-induced SE displayed a reduced induction of GFAP, Iba1 and CD11b mRNA (Fig. 8E). The average seizure-induced upregulation of mRNA for the glial markers together were reduced by almost $60 \% 4$ days after pilocarpine-induced SE $(23 \pm 5$ mean fold induction for vehicle versus $9 \pm 3$ mean fold induction for TG8-260, $p=.02$, paired $t$ test fold change values), suggesting that TG8-260 reduces SE-induced gliosis.

\section{Hippocampal Neurodegeneration Is Largely Unaffected by TG8-260}

Representative coronal sections through the rat hippocampus showed that pilocarpine-induced SE produced robust neurodegeneration that can be detected with FluoroJade histochemistry 4 days later (Fig. 9A, B). Neuronal death was assayed by counting the number of brightly labeled FluoroJade B positive (FJB+) neurons in stained sections throughout the hippocampus. This analysis revealed a large number of degenerating neurons in the hilus and $\mathrm{CA} 1$ and $\mathrm{CA} 3$ regions of the hippocampus of rats that were administered pilocarpine and experienced SE. To determine whether TG8-260 administration alters hippocampal neurodegeneration in pilocarpine-treated rats, FJB positive cells were counted in the hilus, CA1 and CA3 and compared to vehicle-treated rats. Neurodegeneration was detected in all three regions using FluoroJade B. There was no difference in the number of hilar FJB+ neurons per section taken from vehicle-treated rats compared to TG8-260 treated rats $(41 \pm 6$ cells per section for vehicle-treated rats, $n=9$ vs $46 \pm 5$ for TG8-260 treated rats, $n=9$ ) (Fig. 9C). The CA1 displayed a trend for a lower average number of $\mathrm{FJB}+$ cells per section in rats administered TG8-260 compared to vehicle $(231 \pm 32$ $\mathrm{FJB}+$ cells per hippocampal section for vehicle, $n=9$ versus $149 \pm 40$ for TG8-260, $n=9 ; p=.13, t$ test) (Fig. 9D). No neuroprotection was observed in the $\mathrm{CA} 3$ region of TG8-260 treated rats $(82 \pm 23$ FJB+ cells per hippocampal section for vehicle, $n=9$ vs $65 \pm 23$ for TG8- 
Methylscopolamine and Terbutaline $(2.5 \mathrm{mg} / \mathrm{kg}$ each, s.c., in $0.9 \% \mathrm{NaCl}$ )

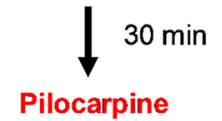

( $380 \mathrm{mg} / \mathrm{kg}$ s.c., in $0.9 \% \mathrm{NaCl}$ )

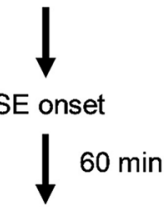

Diazepam (10 $\mathrm{mg} / \mathrm{kg}$, i.p.)<smiles>[13CH3]</smiles>

TG8-260 or vehicle

(2, 8 and $20 \mathrm{hr}$ after SE onset)

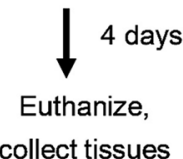

b
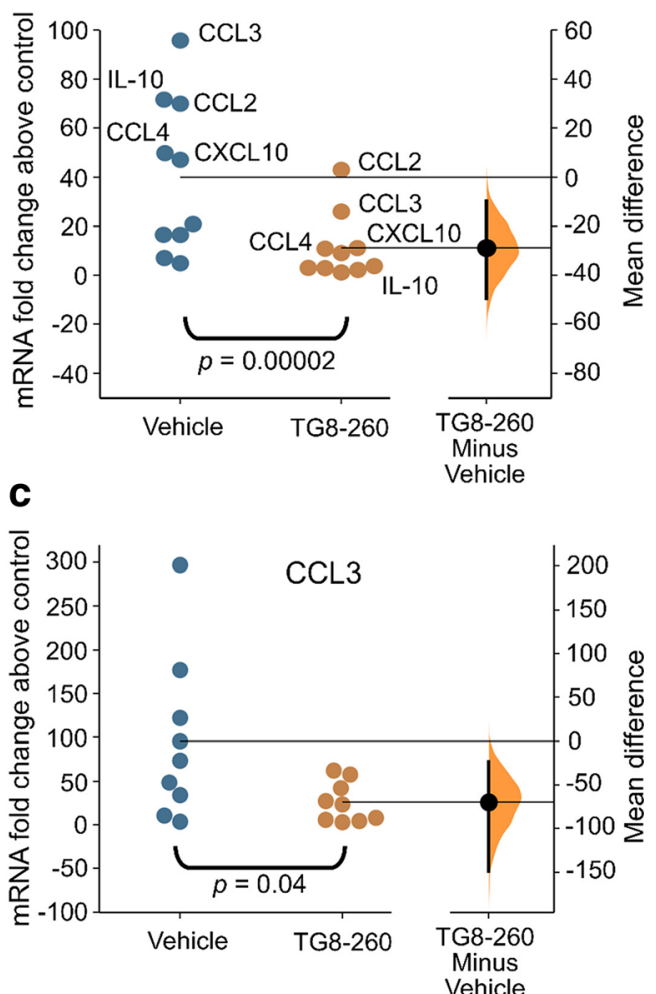

Fig. 5 TG8-260 attenuates hippocampal neuroinflammation. (A) Experimental paradigm of chemical administration in rats. (B) The mean difference between vehicle and TG8-260 is shown in the Gardner-Altman estimation plots. Both groups are plotted on the left axes; the mean difference is plotted on a floating axis on the right as a bootstrap sampling distribution. The mean difference is depicted as a black circle; the $95 \%$ confidence interval is indicated by the ends of the vertical bar. The change in abundance of 10 inflammatory mediator mRNAs from the hippocampus of rats 4 days after injection with pilocarpine to induce status epilepticus is shown. Post-SE treatment was three doses of TG8-

260, $n=9$ ) (Fig. 9E). A comparison of the total number of $\mathrm{FJB}+$ pyramidal cells in the combined CA1 and CA3 regions revealed no significant neuroprotection by TG8-260 (313 $\pm 41 \mathrm{FJB}+$ cells per hippocampal section for vehicle, $n=9$ vs $214 \pm 55$ for TG8-260, $n=9)(p=.17, t$ test $)$. No neurodegeneration was observed in non-seizure control rats that were administered sterile saline instead of pilocarpine (not shown).

\section{TG8-260 Failed to Reduce Blood-Brain Barrier Leakiness after SE}

The blood-brain barrier (BBB) plays a critical role in homeostasis in the brain. One measure of BBB integrity can be determined by perfusing animals with buffered saline to remove blood from the brain, and subsequently measuring the extravasation of serum albumin into the brain parenchyma by western blot. Serum albumin is low in the brain parenchyma when the BBB is intact. In
260 ( $n=9$ rats) or vehicle ( $n=9$ rats). Following pilocarpine-induced SE, the mRNA fold change for all 10 mediators as a group was significantly reduced by TG8-260 compared to vehicle ( $p=.00002$, paired $t$ test on $\Delta \Delta \mathrm{CT}$ values). Data are mean $\pm \mathrm{SEM}$. The other five inflammatory mediators that are not labeled in the figure are: IL- $1 \beta$, IL-6, IL-15, $\mathrm{TNF} \alpha$, and COX-2. (C) Change in mRNA abundance of CCL3 mRNA in the hippocampus of rats administered TG8-260 or vehicle after pilocarpine-induced SE ( $p=.01, t$ test using $\Delta \Delta$ CT values). Each symbol represents a single rat

rats, elevated levels of serum albumin were found in the cortex 4 days after DFP-induced SE [6], which is consistent with breakdown of the BBB. Serum albumin was increased almost 1.6-fold in the cortex of rats that experienced $1 \mathrm{~h}$ of pilocarpine-induced SE measured 4 days later (Fig. 10). Albumin levels appeared higher in the brain parenchyma of rats that received three intraperitoneal injections of TG8-260 delivered 2, 8 and $20 \mathrm{~h}$ after SE onset (Fig. 10) indicating that TG8-260 does not reduce BBB leakiness following pilocarpine-induced SE.

\section{Functional Recovery after SE}

Using a modified Irwin test, we determined the effect of selective EP2 inhibition on a subset of normal behavioral characteristics of rats prior to any injections on day zero and again $24 \mathrm{~h}$ and 4 days after pilocarpine-induced SE. All rats subjected to the modified Irwin test prior to any injections on day zero scored 0 indicative of normal rat health and behavior. 
Fig. 6 Pilocarpine-induced CCL3 upregulation in microglia. Representative fluorescence images showing negative CCL3 immunostaining in the hippocampal CA1 $(\boldsymbol{A})$ and CA3 (B) region of rats that did not experience SE. Positive CCL3 staining was detected in microglia in the CA1 (C) and CA3 (D) regions of the hippocampus $24 \mathrm{~h}$ after pilocarpine-induced SE. The dashed lines represent the principal cell layers in the CA1 and $\mathrm{CA} 3$ regions. The arrows indicate typical positive CCL3 staining. Scale bar is $40 \mu \mathrm{m}(\mathbf{A}-\mathbf{D})$.

$S$. radiatum $=$ stratum radiatum . Positive CCL3 staining was detected in CD11b expressing microglia in the amygdala region $(\mathbf{E})$ $24 \mathrm{~h}$ after pilocarpine-induced SE. The arrows and arrowheads indicate typical positive CCL3 and $\mathrm{CD} 11 \mathrm{~b}$ staining. Scale bar is $10 \mu \mathrm{m}$ in $(\mathbf{E})$
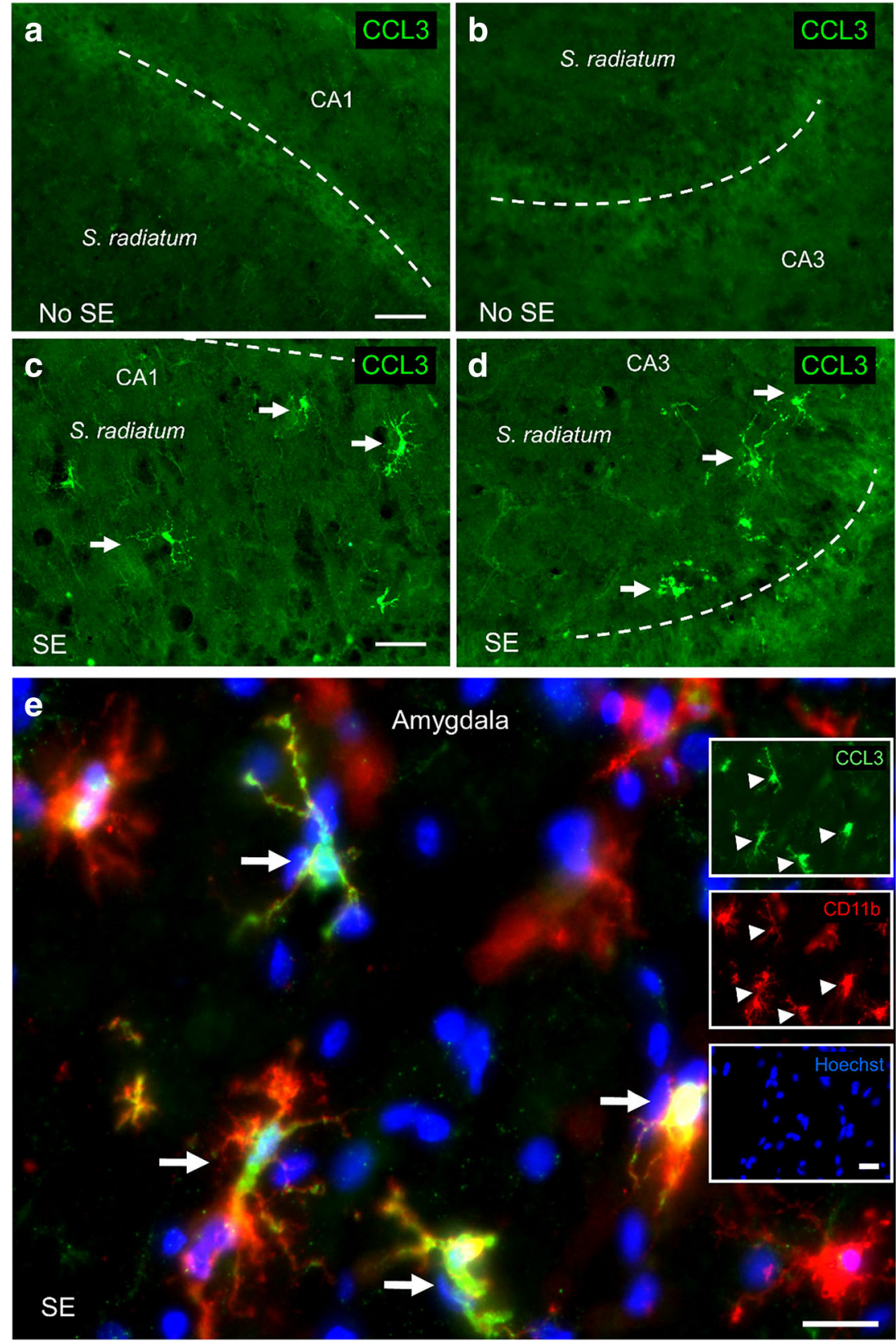

However, a large majority of rats failed (score $\geq 12$ ) the modified Irwin test measured $24 \mathrm{~h}$ after pilocarpine induced SE (Supplemental Fig. 1A), consistent with compromised health. None of the rats that failed the Irwin test showed signs of infection from the multiple injections on day zero, but instead they displayed lethargy. No difference was detected in the 24$\mathrm{h}$ modified Irwin test score of rats that were administered TG8-260 or the vehicle after pilocarpine $(13.3 \pm 0.8$ for vehicle, $n=8 ; 14.7 \pm 0.6$ for TG8-260, $n=7 ; p=.2, t$ test) (Supplemental Fig. 1A). Similarly, no difference was detected in the modified Irwin test score on day 4 of rats that were administered TG8-260 or the vehicle after pilocarpine even though all of the rats recovered (score $\leq 12$ ) suggesting that inhibition of the EP2 receptor by TG6-280 does not alter the 12 physical/behavioral characteristics measured at $24 \mathrm{~h}$ and 4 days after SE.

Rats that experience SE typically lose body weight over the first 2 days and then start to regain weight from day three onward [4-6, 8]. The amount of weight the rats lose appears to correlate with the intensity of the initial SE insult. All rats exposed to pilocarpine lost a similar amount of weight on day 1 ( $\sim 10 \%$ average) (Supplemental Fig. 1B). Rats injected with saline, diazepam and then drug or vehicle that did not experience SE stabilized their weight by day 2 and by day 3 they returned close to their original weight (Supplemental Fig. 1B, black circles, open squares). By contrast, rats that experienced 
a

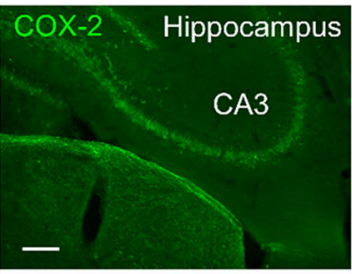

b

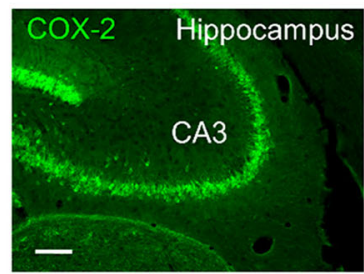

C
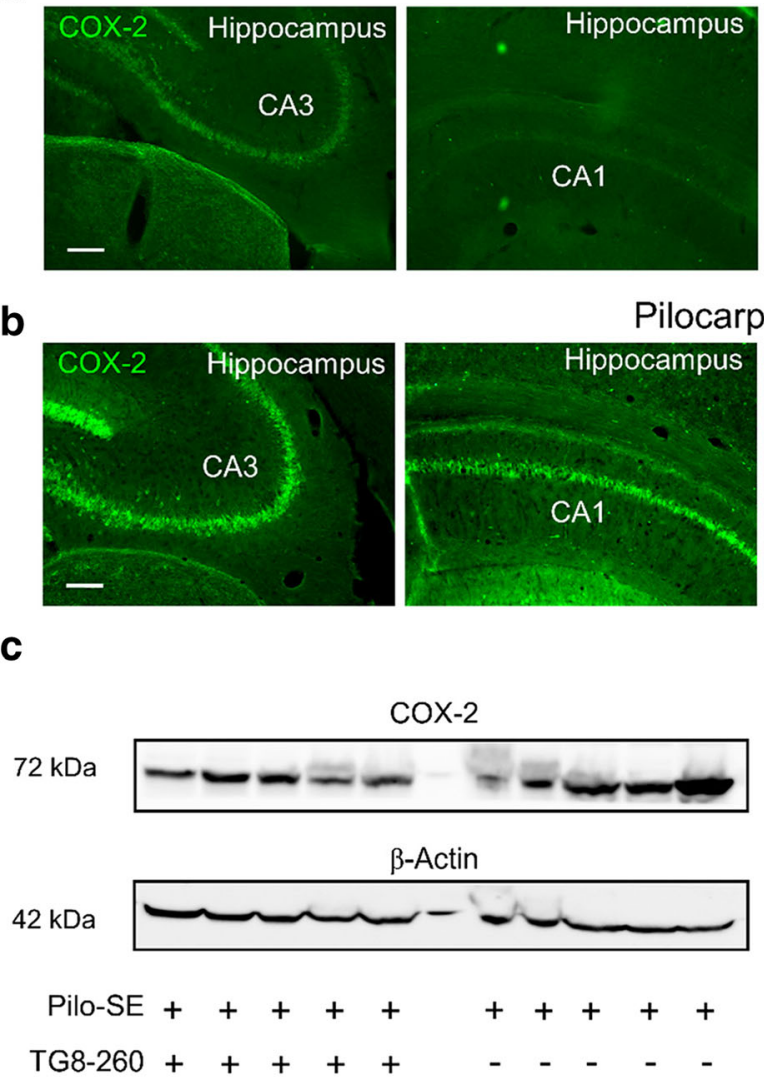

No-SE
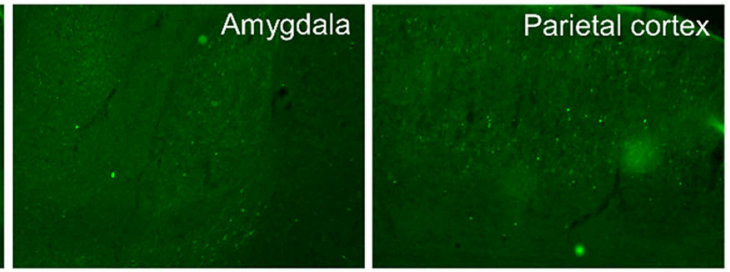

Pilocarpine-SE
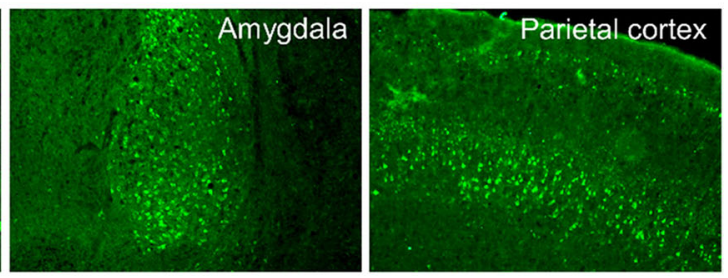

d

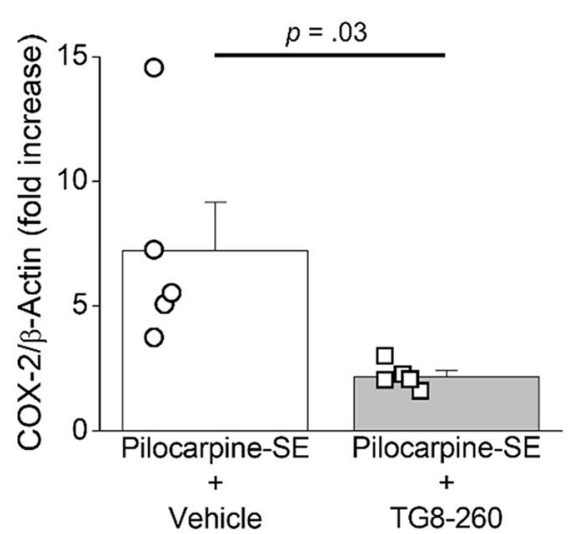

Fig. 7 TG8-260 reduces COX-2 induction in principal neurons following pilocarpine. Fluorescent images taken from the CA3 and CA1 regions in the hippocampus, the amygdala and parietal cortex (50× magnification) reveal basal expression of neuronal COX-2 in rats that did not experience status epilepticus (A) and prominent COX-2 staining (green) in rats $24 \mathrm{~h}$ after pilocarpine-induced SE (B). The images shown are representative of five sections each from three rats. Scale bar, $50 \mu \mathrm{m}$. (C) Western blot of COX-2 protein levels (bands in the top box) on day 4 in the cortex of rats that experienced SE induced by pilocarpine followed by administration of

SE continued to lose weight on day 2 regardless of whether they were administered TG8-260 or the vehicle (Supplemental Fig. 1B).

\section{Discussion}

Here, we used a pilocarpine model of status epilepticus (SE) in adult rats and investigated the efficacy of a second-generation small-molecule EP2 antagonist, TG8-260, to attenuate SE associated neuropathology. As observed with other chemoconvulsants, peripheral administration of a high dose of pilocarpine rapidly resulted in seizures and SE, which caused a rapid and robust inflammatory reaction in the brain characterized by the increased expression of a number of inflammatory mediators. Neurodegeneration in seizure sensitive limbic brain regions, gliosis and loss of blood-brain barrier

(BBB) integrity were also present in the brains of rats administered pilocarpine. A good time point for effectively quantifying neuroinflammation, neurodegeneration, gliosis and break down of the BBB in rodent models of SE is 4 days after SE $[1-4,6,8]$. Our major finding is that TG8-260 replicates the anti-inflammatory effects of the earlier generation EP2 antagonist, TG6-10-1, but not the neuroprotective or bloodbrain barrier sparing effects, which points to neuroinflammation as a possible target of EP2 receptors.

Prior to administering TG8-260 to animals, we investigated its in vitro inflammation modulating properties and found that TG8-260 reverses the change in expression of inflammatory mediators induced by the combination of LPS and EP2 activation, consistent with other EP2 receptor antagonists created in our laboratory $[1,2,21,28,29]$. Although IL-10 displays pleiotropic effects it is largely an anti-inflammatory mediator and therefore enhancing IL-10 expression will quell 

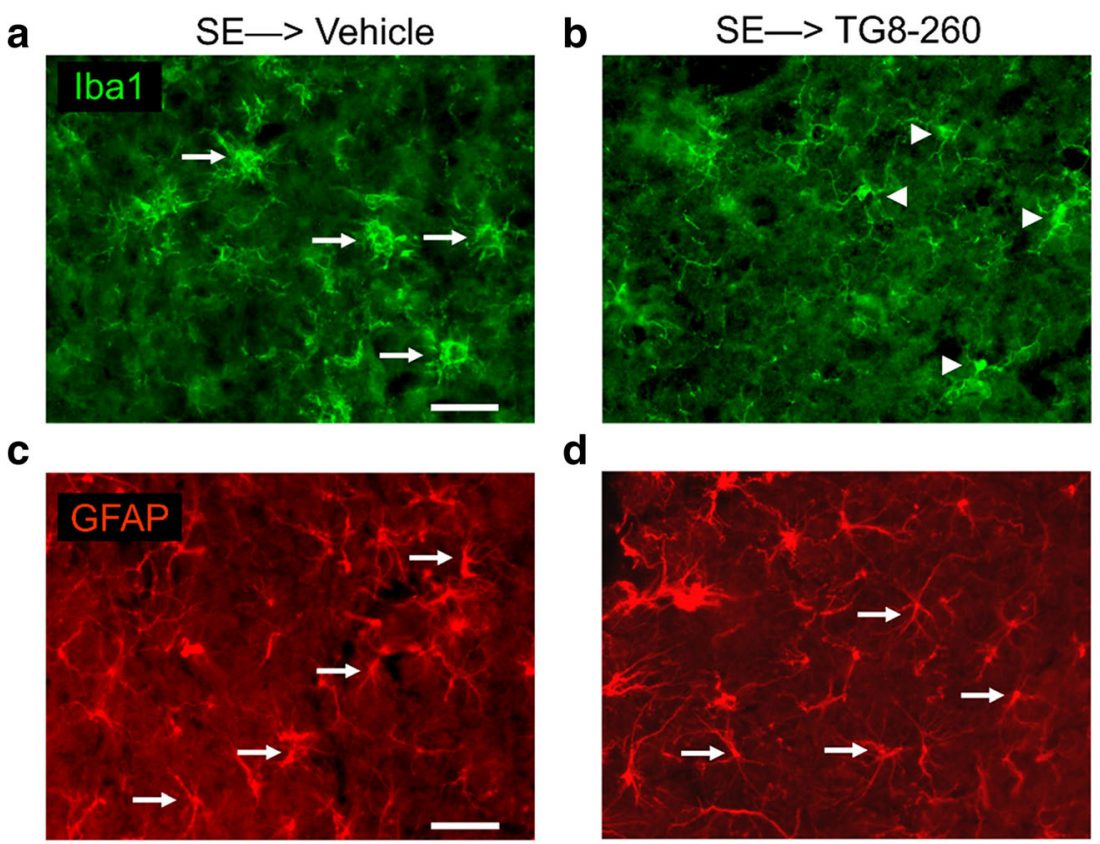

d

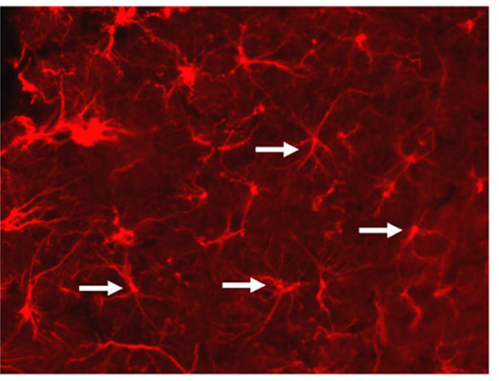

e

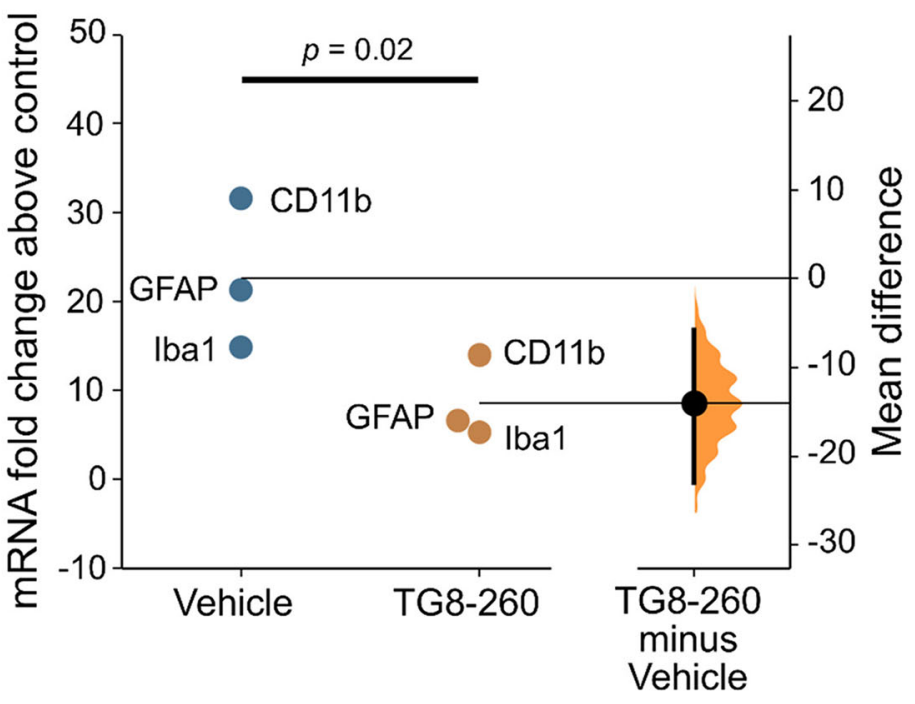

Fig. 8 TG8-260 blunts hippocampal microgliosis. Representative fluorescence images $(100 \times$ total magnification) taken from the hippocampal CA3 region showing positive Ibal immunostaining (green) as a microglial marker in vehicle (A) and TG8-260 (B) injected rats 4 days after pilocarpine-induced SE as well as GFAP immunostaining (red) as an astrocyte marker in vehicle (C) and TG8-260 (D) injected rats. Four days after pilocarpine-induced status epilepticus, microgliosis was obvious in the sections obtained from rats administered vehicle as defined by the increased size of the cell body and amoeboid appearance (short and thick processes). The arrows in panel A indicate typical activated microglia. The arrowheads in panel B indicate typical resting microglia. Astrogliosis was not as obvious. The arrows in panels C and D

inflammation. Similarly, chemokines secreted by microglia recruit other cells to injured tissue to facilitate healing and restoration. Therefore, in the simplified cell culture system incubation of the BV2 microglia with TG8-260 produced an anti-inflammatory effect as it enhanced expression of IL-10 and the chemokines (CCL3 and CCL4) in the presence of a

indicate typical resting astrocytes. Scale bar, $20 \mu \mathrm{m}$. (E) the mean difference between vehicle and TG8-260 is shown in the Gardner-Altman estimation plot. The mean difference is plotted on a floating axis on the right as a bootstrap sampling distribution. Change in abundance of three glial marker mRNAs from the hippocampus of rats 4 days after injection with pilocarpine to induce status epilepticus. Post-SE treatment was three doses of TG8-260 ( $n=9$ rats) or vehicle ( $n=9$ rats). Following pilocarpine-induced status epilepticus, the mRNA fold change for the three glial markers as a group was significantly reduced by TG8-260 compared to vehicle ( $p=.02$, paired $t$ test on fold change values). The symbols are the average fold change of 9 rats for each glial marker

combination of LPS and a selective EP2 agonist. Nevertheless, the BV2-hEP2 cell culture is a homogeneous population useful for deciphering the role EP2 in microglial function. Exposure to bacterial LPS results in an immunogenic inflammatory response, whereas following SE in vivo other cell types such as neurons contribute to neurogenic 
a

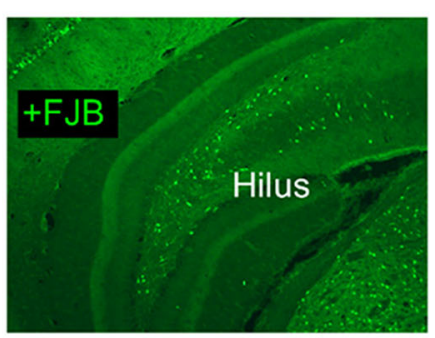

b

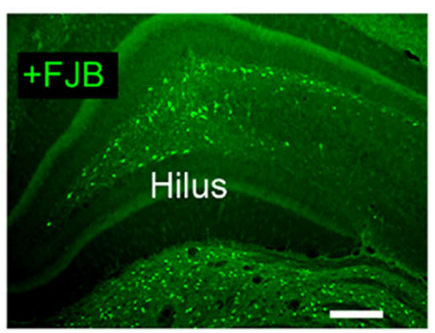

C

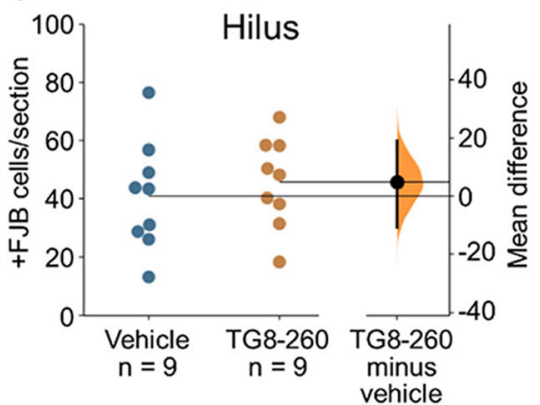

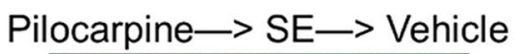
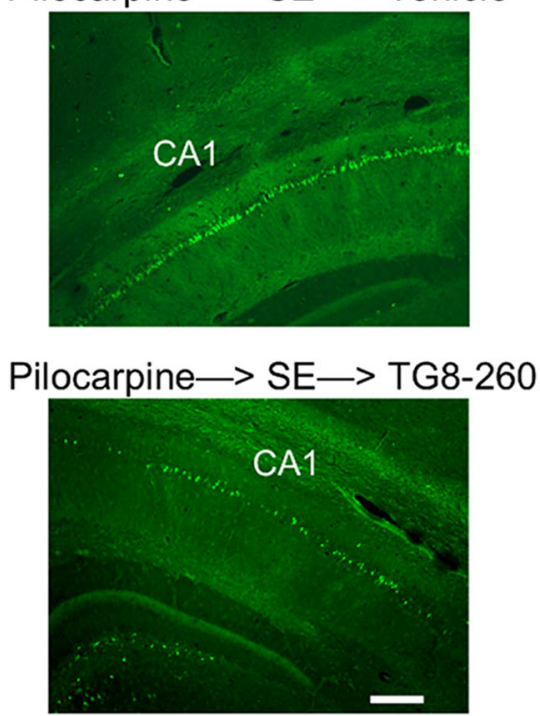

d

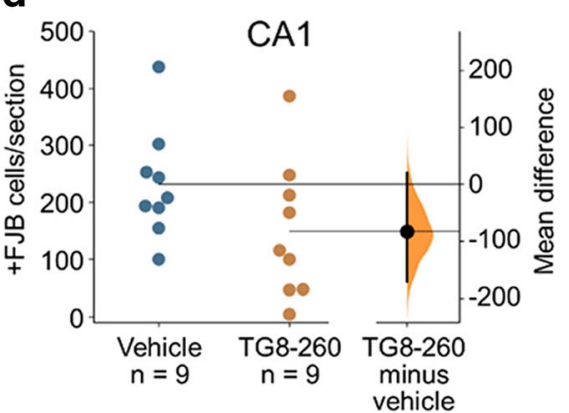

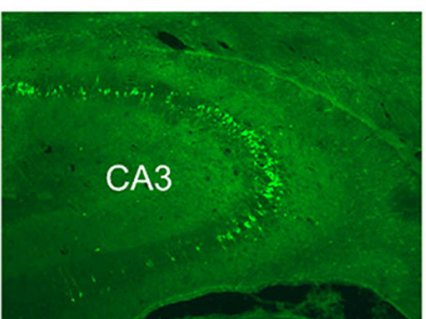

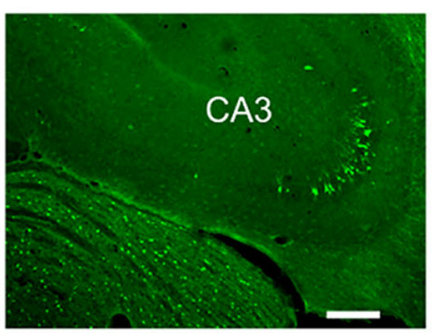

e

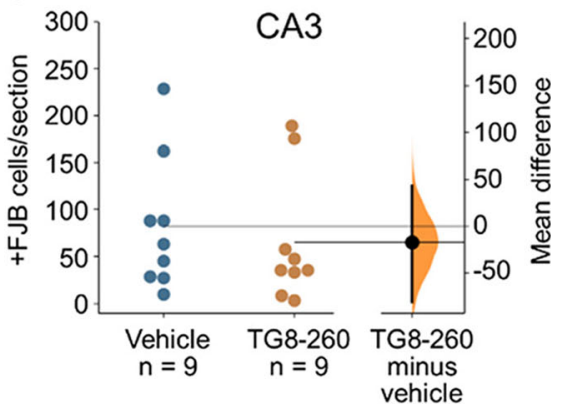

Fig. 9 Neurodegeneration following pilocarpine-induced status epilepticus. Representative images of FluoroJade B staining in hippocampal sections $(40 \mu \mathrm{m})$ in the hilus, CA1 and CA3 regions for rats treated with vehicle (A) 4 days after pilocarpine-induced status epilepticus and rats injected with three doses of TG8-260 (B). Images were taken at a total magnification of $\times 50$. The images are representative of 8 sections per rat. Scale bar, $100 \mu \mathrm{m}$. The mean difference in the number of injured neurons between vehicle and TG8-260 treated rats that experienced pilocarpineinduiced SE is shown in the Gardner-Altman estimation plots for FluoroJade B positive cells (FJB+) in the hilus (C), CA1 (D), and CA3 (E). In each plot both groups are plotted on the left axes; the mean difference is plotted on a floating axes on the right as a bootstrap sampling distribution. The mean difference is depicted as a dot; the $95 \%$ confidence interval is indicated by the ends of the vertical bar inflammation. Therefore, the anti-inflammatory effects of TG8-260 in BV2 microglia following activation might differ from the effects seen in the brain of an animal following SE. The contribution of EP2 activation in various cell types in vivo should be considered when comparing the model systems. There is growing evidence that SE-induced neuroinflammation in rats precedes a delayed phase of neurodegeneration, gliosis and breakdown of the blood-brain barrier [4, 7]. This is important when assaying for the effectiveness of a novel compound as the efficacy may depend on the manifestation of the pathology.

Previous investigation of broader neuroinflammatory signaling in rodents following SE informed and helped us to select the neuroinflammatory pathway in the current study. COX-2 is a key inflammatory mediator in the neuroinflammatory response in the brain following the onset of SE. We previously demonstrated that EP2 antagonism by the first generation antagonist (TG6-10-1) following SE in rodents recapitulates the features observed following selective COX-2 inhibition after SE $[1-4,6]$. Furthermore, the EP2 receptor is expressed on the surface of microglia and regulates the microglial inflammatory response following activation by seizures or LPS $[30,31]$. Therefore, the neuroinflammatory pathway we chose to focus on in the current study is directly related to COX-2 signaling and microglial activation. The results of the hippocampal expression pattern of the 10 inflammatory mediators investigated on day 4 suggest that inhibition of the EP2 receptor using TG8-260 is strongly anti-inflammatory in animals. Two key inflammatory mediators (CCL3 and COX-2) were closely examined. CCL3 is a chemokine that attracts immune cells to sites of injury, and it is induced following NMDA-evoked neuronal injury in cultured rat corticostriatal slices [32]. The 

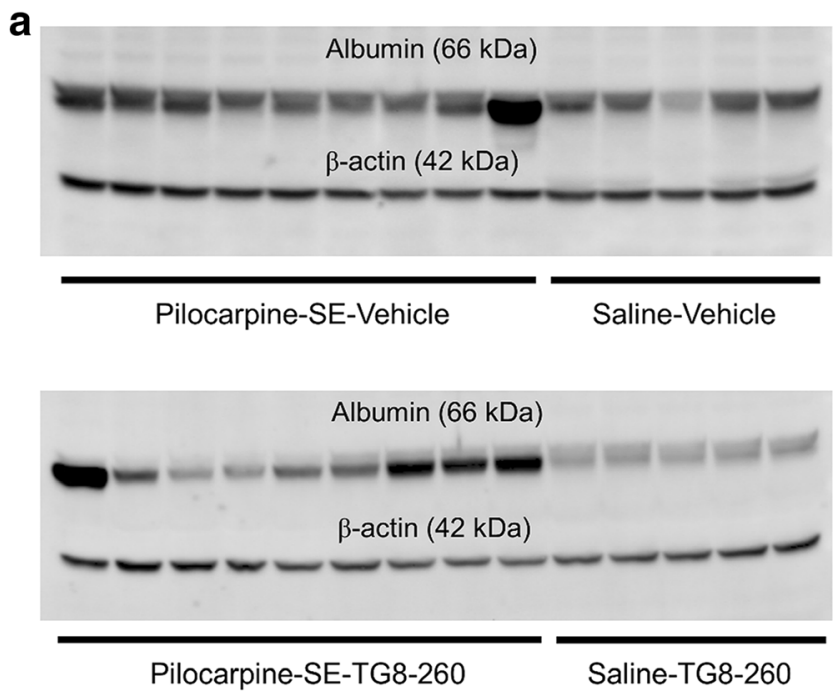

b

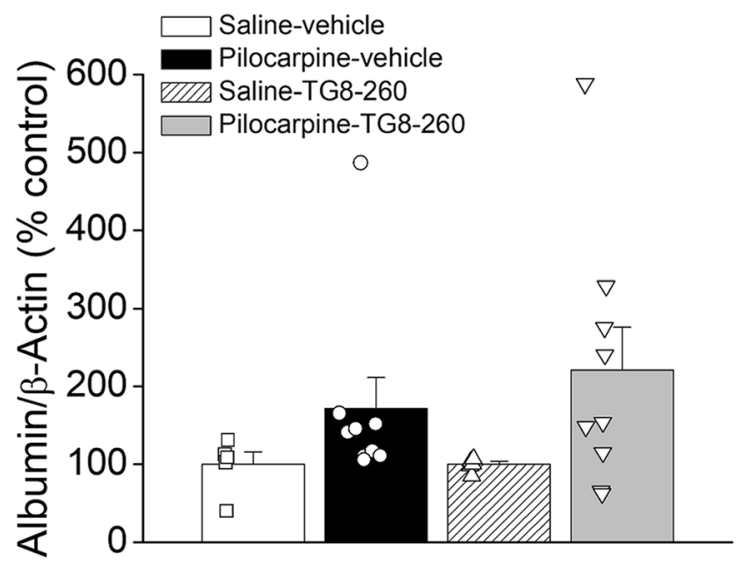

Fig. 10 Blood-brain barrier leakiness is unaffected by TG8-260. (A) the amount of serum albumin in the cortex 4 days after SE was used to assess the integrity of the blood-brain barrier as all rats were perfused with sterile saline to remove blood from the brain. The albumin levels (black bands, below) in the cortex of non-seizure controls or rats that experienced $60 \mathrm{~min}$ of SE induced by pilocarpine that received TG8-260 (25 mg/kg, i.p. $)$ or the vehicle (5\% NMP, $5 \%$ solutol-HS15 and $90 \%$ saline) at 2,8 and $20 \mathrm{~h}$ after SE onset were measured by western blot with $\beta$-actin (bottom bands) used as a loading control. (B) the band intensity of the albumin was normalized to the housekeeping $\beta$-actin. The albumin level was similar for TG8-260 and vehicle-treated rats after SE $[171 \pm 40 \%$ of control for vehicle-treated rats $(n=9)$ versus $221 \pm 55 \%$ of control for TG8-260 injected rats $(n=9)]$. The data are mean \pm standard error of the mean. The symbols represent each individual rat within the group. $p>0.05$, one-way ANOVA with post hoc Bonferroni test with selected pairs (comparing pilocarpine-vehicle $v s$ pilocarpine-TG8-260)

increase in CCL3 mRNA found here is consistent with CCL3 induction that would be expected to help recruit monocytes after pilocarpine-induced SE in a similar manner as shown following cerebral hypoxic-ischemic injury [33]. The strong induction of CCL3 in the hippocampus was consistent with intense positive staining for CCL3 in microglia in seizure sensitive brain regions $24 \mathrm{~h}$ after SE (Fig. 6C-E). It is unclear whether disruption of CCL3 signaling in the brain alters neuroinflammation. However, in the current study we have shown that inhibition of EP2 reduced CCL3 levels in the hippocampus.

COX-2 is the rate-limiting enzyme in the production of prostaglandins and is a key mediator of inflammation in seizure disorders as discussed in a recent review [11]. Similar to CCL3, COX-2 induction in the hippocampus is very prominent $24 \mathrm{~h}$ following pilocarpine administration (Fig. 7B). However, unlike CCL3 COX-2 is induced in neurons. Data presented here in the rat pilocarpine model confirm those in the mouse pilocarpine model [2], the rat DFP model [4, 8] and the rat soman model [34], all demonstrating that induction of COX-2 persists for several days after SE. Consistent with an anti-inflammatory effect of EP2 inhibition following SE, TG8-260 significantly blunted COX-2 expression 4 days following SE. This is consistent with our previous demonstration that administration of another EP2 receptor antagonist (TG610-1) after SE blunts the expression of COX-2 [2, 4, 6]. Furthermore, the data presented here on the efficacy of TG8260 to attenuate inflammation strengthens the conclusion that EP2 inhibition recapitulates the anti-inflammatory effect of COX-2 inhibition in rodent models of SE.

Quantification of the mRNA for the glial markers (Iba1 and GFAP) 4 days after pilocarpine-induced SE revealed a reduction of GFAP and Ibal in rats administered three doses of TG8-260 coinciding with a decrease in mRNA levels of the inflammatory mediators. Microgliosis, but not astrogliosis, defined by the amoeboid appearance of the cells, was prominent in the brain of rats administered the vehicle 4 days after pilocarpine-induced SE as shown in representative images in Fig. 8A-D. Similar to TG6-10-1 treatment in rats exposed to DFP [4] and mice that experienced pilocarpine-induced SE [2], TG8-260 attenuated cell swelling of microglia, but not astrocytes. This selective effect of EP2 antagonists on microgliosis is attributed to the expression of functional EP2 receptors in microglia [30, 31, 35-39].

Based on the in vitro efficacy of TG8-260 with a Schild $K_{\mathrm{B}}$ of $13.2 \mathrm{nM}$ for human EP2 and the PK profile, and the assumption that pharmacokinetics of TG6-280 are similar in rats and mice, the dosing paradigm for the treatment of rats that experience $S E$ would have maintained plasma and brain levels of TG8-260 above the Schild $K_{\mathrm{B}}$ value for EP2 for a minimum $24 \mathrm{~h}$. However, even with prolonged levels above the EP2 $K_{\mathrm{B}}$ value TG8-260, unlike TG6-10-1, failed to protect hippocampal neurons. It is not clear why TG8-260 is not more neuroprotective because the EP2 $K_{\mathrm{B}}$ value is lower for TG8-260. The low brain:plasma ratio for TG8-260 (0.02), could contribute to the lack of neuroprotection. Perhaps a shorter exposure or different exposure profile is needed to protect neurons as neurodegeneration continues to increase in the days following pilocarpine-induced SE. One important difference between TG8-260 and TG6-10-1 is that TG8-260 inhibits drug metabolizing enzymes CYP3A4 and CYP2C19 (to be reported 
elsewhere). It is known that diazepam is metabolized mainly by CYP3A4 and CYP2C19 to an active metabolite, desmethyldiazepam. This suggests that rats that were administered TG8-260 following SE may have experienced a long lasting sedative effect of diazepam, however we did not notice any excessive sedation in these rats.

Another difference between TG8-260 and TG6-10-1 is that TG8-260 failed to maintain the integrity of the BBB comprised of endothelial cells, astrocytes, pericytes, and microglia $[40,41]$. This suggests that the reduction of neuroinflammation observed in the hippocampus does not necessarily translate to an effective attenuation of breakdown of the BBB. However, it is important to mention here that a 1.6-fold increase in serum albumin levels (Fig. 10) does not represent as large a breach of the BBB in vehicle-treated rats as we had seen previously in mice and rats. For example, rats that experience DFP-induced SE display a 4-fold increase in the level of serum albumin in the cortex as measured on day 4 after SE. Perhaps following pilocarpine-induced SE, breakdown of the BBB occurs earlier than observed following DFP. Additionally, the dark albumin bands detected in the western blot for saline-treated rats administered the vehicle might reflect an incomplete perfusion, which in the quantification analysis could reduce the fold change compared with control saline-treated rats. The incomplete perfusion of saline-vehicle rats might have prevented the detection of a beneficial effect of TG8-260 on BBB integrity. In the future, it will be important to investigate the time-dependent breakdown of the BBB following pilocarpine-induced $\mathrm{SE}$ in rats and determine whether EP2 inhibition by TG8-260 protects the BBB following complete removal of blood from the brain parenchyma.

\section{Conclusion}

Administration of pilocarpine results in SE-induced neuropathology including a robust inflammatory response, hippocampal neurodegeneration, hippocampal gliosis and breakdown of the blood-brain barrier in adult rats. The EP2 antagonist, TG8-260, attenuated hippocampal neuroinflammation and gliosis within 4 days after pilocarpine administration. We conclude that delayed inhibition of EP2 receptors by TG8-260 administered beginning $2 \mathrm{~h}$ after SE results in beneficial consequences. Among all of the beneficial effects of EP2 blockade, the anti-inflammatory effect and attenuated microgliosis are the most consistent across EP2 antagonists, dosing routes, species, and models of SE. Together, these studies give insight and reveal the effectiveness of EP2 inhibition to combat SEinduced neuropathology. It will be important to determine whether inhibition of neuroinflammation and gliosis by TG8-260 treatment leads to reduced SE-induced long-term cognitive deficits in rodents.
Supplementary Information The online version contains supplementary material available at https://doi.org/10.1007/s13311-020-00969-5.

Acknowledgments We thank Ms. Wenyi Wang and Dr. Damoder Reddy Motati (Emory University School of Medicine, Atlanta, GA) for their helpful technical discussions. We thank ONO Pharmaceuticals for kindly providing ONO-AE1-259-1. This work was supported by the NIH/NIA grant U01 AG052460 (TG), NINDS grants R21/R33 NS101167 (TG), RO1 NS097776 and R01 NS112308 (RD).

Required Author Forms Disclosure forms provided by the authors are available with the online version of this article.

\section{References}

1. Jiang J, Ganesh T, Du Y, Quan Y, Serrano G, Qui M, et al. Small molecule antagonist reveals seizure-induced mediation of neuronal injury by prostaglandin E2 receptor subtype EP2. Proc Natl Acad Sci U S A 2012;109(8):3149-54.

2. Jiang J, Quan Y, Ganesh T, Pouliot WA, Dudek FE, Dingledine R. Inhibition of the prostaglandin receptor EP2 following status epilepticus reduces delayed mortality and brain inflammation. Proc Natl Acad Sci U S A 2013;110(9):3591-6.

3. Jiang J, Yang MS, Quan Y, Gueorguieva P, Ganesh T, Dingledine R. Therapeutic window for cyclooxygenase-2 related antiinflammatory therapy after status epilepticus. Neurobiol Dis 2015;76:126-36.

4. Rojas A, Ganesh T, Lelutiu N, Gueorguieva P, Dingledine R. Inhibition of the prostaglandin EP2 receptor is neuroprotective and accelerates functional recovery in a rat model of organophosphorus induced status epilepticus. Neuropharmacology 2015;93: 15-27.

5. Rojas A, Ganesh T, Manji Z, O'Neill T, Dingledine R. Inhibition of the prostaglandin E2 receptor EP2 prevents status epilepticusinduced deficits in the novel object recognition task in rats. Neuropharmacology 2016;110(Pt A):419-30.

6. Rojas A, Ganesh T, Wang W, Wang J, Dingledine R. A rat model of organophosphate-induced status epilepticus and the beneficial effects of EP2 receptor inhibition. Neurobiol Dis 2020;133:104399.

7. Rojas A, Wang J, Glover A, Dingledine R. Urethane attenuates early neuropathology of diisopropylfluorophosphate-induced status epilepticus in rats. Neurobiol Dis 2020;140:104863.

8. Rojas A, Wang W, Glover A, Manji Z, Fu Y, Dingledine R. Beneficial Outcome of Urethane Treatment Following Status Epilepticus in a Rat Organophosphorus Toxicity Model. eNeuro 2018;5(2).

9. Serrano GE, Lelutiu N, Rojas A, Cochi S, Shaw R, Makinson CD, et al. Ablation of cyclooxygenase- 2 in forebrain neurons is neuroprotective and dampens brain inflammation after status epilepticus. J Neurosci 2011;31(42):14850-60.

10. Dingledine R, Coulter DA, Fritsch B, Gorter JA, Lelutiu N, McNamara J, et al. Transcriptional profile of hippocampal dentate granule cells in four rat epilepsy models. Sci Data 2017;4:170061.

11. Rojas A, Chen D, Ganesh T, Varvel NH, Dingledine R. The COX2/prostanoid signaling cascades in seizure disorders. Expert Opin Ther Targets 2019;23(1):1-13.

12. Rojas A, Jiang J, Ganesh T, Yang MS, Lelutiu N, Gueorguieva P, et al. Cyclooxygenase-2 in epilepsy. Epilepsia 2014;55(1):17-25.

13. Jiang J, Yu Y, Kinjo ER, Du Y, Nguyen HP, Dingledine R. Suppressing pro-inflammatory prostaglandin signaling attenuates excitotoxicity-associated neuronal inflammation and injury. Neuropharmacology 2019;149:149-60. 
14. Racine RJ. Modification of seizure activity by electrical stimulation. II. Motor seizure. Electroencephalogr Clin Neurophysiol 1972;32(3):281-94.

15. Irwin S. Comprehensive observational assessment: Ia. A systematic, quantitative procedure for assessing the behavioral and physiologic state of the mouse. Psychopharmacologia 1968;13(3):222-57.

16. Schmued LC, Albertson C, Slikker W, Jr. Fluoro-Jade: a novel fluorochrome for the sensitive and reliable histochemical localization of neuronal degeneration. Brain Res 1997;751(1):37-46.

17. Paxinos G, Watson C. The rat brain in stereotaxic coordinates. 2nd. Sydney: Academic Press; 1986. xxvi, 237 p. of plates p.

18. Nelson TE, Gruol DL. The chemokine CXCL10 modulates excitatory activity and intracellular calcium signaling in cultured hippocampal neurons. J Neuroimmunol 2004;156(1-2):74-87.

19. Sato Y, Ohshima T, Kondo T. Regulatory role of endogenous interleukin-10 in cutaneous inflammatory response of murine wound healing. Biochem Biophys Res Commun 1999;265(1): 194-9.

20. Livak KJ, Schmittgen TD. Analysis of relative gene expression data using real-time quantitative PCR and the 2(-Delta Delta C(T)) Method. Methods 2001;25(4):402-8.

21. Rojas A, Banik A, Chen D, Flood K, Ganesh T, Dingledine R. Novel Microglia Cell Line Expressing the Human EP2 Receptor. ACS Chem Neurosci 2019;10(10):4280-92.

22. Ganesh T, Jiang J, Dingledine R. Development of second generation EP2 antagonists with high selectivity. Eur J Med Chem 2014;82:521-35.

23. Ganesh T, Jiang J, Yang MS, Dingledine R. Lead optimization studies of cinnamic amide EP2 antagonists. J Med Chem 2014;57(10):4173-84.

24. Ho J, Tumkaya T, Aryal S, Choi H, Claridge-Chang A. Moving beyond P values: data analysis with estimation graphics. Nat Methods 2019;16(7):565-6.

25. Borges K, McDermott DL, Dingledine R. Reciprocal changes of CD44 and GAP-43 expression in the dentate gyrus inner molecular layer after status epilepticus in mice. Exp Neurol 2004;188(1):1-10.

26. Frank-Cannon TC, Alto LT, McAlpine FE, Tansey MG. Does neuroinflammation fan the flame in neurodegenerative diseases? Mol Neurodegener 2009;4:47.

27. Kielian T. Neuroinflammation: good, bad, or indifferent? J Neurochem 2014;130(1):1-3.

28. Amaradhi R, Banik A, Mohammed S, Patro V, Rojas A, Wang W, et al. Potent, Selective, Water Soluble, Brain-Permeable EP2 Receptor Antagonist for Use in Central Nervous System Disease Models. J Med Chem 2020;63(3):1032-50.

29. Ganesh T, Banik A, Dingledine R, Wang W, Amaradhi R. Peripherally Restricted, Highly Potent, Selective, AqueousSoluble EP2 Antagonist with Anti-Inflammatory Properties. Mol Pharm 2018;15(12):5809-17.
30. Fu Y, Yang MS, Jiang J, Ganesh T, Joe E, Dingledine R. EP2 Receptor Signaling Regulates Microglia Death. Mol Pharmacol 2015;88(1):161-70.

31. Quan Y, Jiang J, Dingledine R. EP2 receptor signaling pathways regulate classical activation of microglia. J Biol Chem 2013;288(13):9293-302.

32. Okamura T, Katayama T, Obinata C, Iso Y, Chiba Y, Kobayashi H, et al. Neuronal injury induces microglial production of macrophage inflammatory protein-1alpha in rat corticostriatal slice cultures. J Neurosci Res 2012;90(11):2127-33.

33. Cowell RM, Xu H, Galasso JM, Silverstein FS. Hypoxic-ischemic injury induces macrophage inflammatory protein-1alpha expression in immature rat brain. Stroke 2002;33(3):795-801.

34. Angoa-Perez M, Kreipke CW, Thomas DM, Van Shura KE, Lyman M, McDonough JH, et al. Soman increases neuronal COX-2 levels: possible link between seizures and protracted neuronal damage. Neurotoxicology 2010;31(6):738-46.

35. Bonfill-Teixidor E, Otxoa-de-Amezaga A, Font-Nieves M, SansFons MG, Planas AM. Differential expression of E-type prostanoid receptors 2 and 4 in microglia stimulated with lipopolysaccharide. J Neuroinflammation 2017;14(1):3.

36. Johansson JU, Pradhan S, Lokteva LA, Woodling NS, Ko N, Brown HD, et al. Suppression of inflammation with conditional deletion of the prostaglandin E2 EP2 receptor in macrophages and brain microglia. J Neurosci 2013;33(40):16016-32.

37. Johansson JU, Woodling NS, Brown HD, Wang Q, Andreasson KI. Microarray analysis of the in vivo response of microglia to Abeta peptides in mice with conditional deletion of the prostaglandin EP2 receptor. Genom Data 2015;5:268-71.

38. Li P, Lu J, Kaur C, Sivakumar V, Tan KL, Ling EA. Expression of cyclooxygenase-1/-2, microsomal prostaglandin-E synthase-1 and E-prostanoid receptor 2 and regulation of inflammatory mediators by PGE(2) in the amoeboid microglia in hypoxic postnatal rats and murine BV-2 cells. Neuroscience 2009;164(3):948-62.

39. Li X, Melief E, Postupna N, Montine KS, Keene CD, Montine TJ. Prostaglandin E2 receptor subtype 2 regulation of scavenger receptor CD36 modulates microglial Abeta42 phagocytosis. Am J Pathol 2015;185(1):230-9.

40. Liebner S, Dijkhuizen RM, Reiss Y, Plate KH, Agalliu D, Constantin G. Functional morphology of the blood-brain barrier in health and disease. Acta Neuropathol 2018;135(3):311-36.

41. Sa-Pereira I, Brites D, Brito MA. Neurovascular unit: a focus on pericytes. Mol Neurobiol 2012;45(2):327-47.

Publisher's Note Springer Nature remains neutral with regard to jurisdictional claims in published maps and institutional affiliations. 